\title{
Optimal auditing and insurance in a dynamic model of tax compliance
}

\author{
B. RAVIKUMAR \\ Research Division, Federal Reserve Bank of St. Louis \\ YuZHE ZHANG \\ Department of Economics, Texas A\&M University
}

\begin{abstract}
We study the optimal auditing of a taxpayer's income in a dynamic principalagent model of hidden income. Taxpayers in our model initially have low income and stochastically transit to high income that is an absorbing state. A low-income taxpayer who transits to high income can underreport his true income and evade his taxes. With a constant absolute risk-aversion utility function and a costly and imperfect auditing technology, we show that the optimal auditing mechanism in our model consists of cycles. Within each cycle, a low-income taxpayer is initially unaudited, but if the duration of low-income reports exceeds a threshold, then the auditing probability becomes positive. That is, the tax authority guarantees that the taxpayer is not audited until the threshold duration is reached. We also find that auditing becomes less frequent if the auditing cost is higher or if the variance of income is lower.

KeyworDs. Tax auditing, tax compliance, optimal taxation, stochastic costly state verification.
\end{abstract}

JEL CLASSIFICATION. D82, D86, J65.

\section{INTRODUCTION}

There is a large literature on tax compliance following the approach to crime and punishment developed in Becker (1968) and Stigler (1970). For instance, Reinganum and Wilde $(1985,1986)$ examine a static model where taxpayers' incomes are private information. Using the costly state verification framework developed by Townsend (1979), they study optimal verification schemes when the tax and the penalty are exogenously

B. Ravikumar: b.ravikumar@wustl .edu

Yuzhe Zhang: yuzhe-zhang@econmail . tamu .edu

We are grateful to two anonymous referees whose comments on a previous version of this paper helped us revise the paper substantially. We are also grateful to Roc Armenter, V. V. Chari, April Franco, David Fuller, Hari Govindan, Larry Jones, Marek Kapicka, Ayca Kaya, Narayana Kocherlakota, Igor Livshits, Matt Mitchell, Chris Phelan, Latchezar Popov, Erwan Quintin, Shouyong Shi, Galina Vereschagina, Gustavo Ventura, Cheng Wang, and seminar participants in the Canadian Macroeconomics Study Group 2009 for their helpful comments. The views expressed in this article are those of the authors and do not necessarily reflect the views of the Federal Reserve Bank of St. Louis or the Federal Reserve System.

Copyright $\odot 2012$ B. Ravikumar and Yuzhe Zhang. Licensed under the Creative Commons AttributionNonCommercial License 3.0. Available at http: //econtheory . org.

DOI: $10.3982 /$ TE737 
specified. In this paper, we characterize the optimal auditing and taxation schemes in a dynamic stochastic costly state verification environment.

We develop a model where the tax authority (principal) is risk neutral and taxpayers (agents) have constant absolute risk-averse preferences. Each agent knows his own income but it is unobserved by the principal. The principal may audit an agent to verify his income, but this is costly and imperfect. The tax authority designs an optimal taxation scheme as well as an optimal auditing scheme to maximize the present value of revenue net of audit cost. Taxpayers in our model initially have low income and receive stochastic opportunities each period to transit to high income. For convenience, we assume that high income is an absorbing state. Since income is private information, the taxpayer could conceal the fact that he has transited to high income and evade taxes. We use a dynamic mechanism-design approach to search for the best tax system and auditing system within a large family of state contingent contracts.

Our model contains persistent private information and, as demonstrated by Fernandes and Phelan (2000), the principal's problem contains two state variables: the continuation utility for an agent who just transited to high income and the continuation utility for a low-income agent. We follow Zhang (2009) and set up the principal's problem in continuous time. We then formulate the Hamilton-Jacobi-Bellman equation and use it to study the dynamic behavior of continuation utilities.

Since high income is an absorbing state in our model, the treatment of the agent who transited to high income is straightforward-constant consumption forever and, hence, constant continuation utility. Furthermore, he is never audited. However, the principal has to provide incentives for the low-income agent to truthfully report the transition to high income. Since income is private information, the principal does not fully insure the low-income agent. The distortion in the consumption path for a low-income agent is a key object of interest. We measure this distortion as the difference between the cost of providing the continuation utility to the low-income agent and the cost of providing the same utility using a perfectly smooth consumption path. We show that the distortion is determined by the ratio of the two state variables noted above.

The auditing technology in our model is imperfect: there is an auditing error that labels a low-income agent as having high income. ${ }^{1}$ Our main result is that it is optimal for the principal to audit the agent periodically. The auditing mechanism in our model consists of cycles. The low-income agent could be in one of two states: (i) not audited or (ii) randomly drawn to be audited. Within each cycle, a low-income agent is initially in the not-audited state. He is moved into the random audit state if the duration of his low-income reports exceeds a threshold $N$, where $N$ is pinned down by the primitives of the model. If he is randomly drawn to be audited, then he is moved to the not-audited state after the audit, and a new cycle begins. If a low-income agent is mislabeled as having high income, he is mistakenly punished and continues to stay in the random audit state. While auditing is stochastic, the threshold duration $N$ is not. Put differently, within each cycle the principal guarantees that the agent is not audited until the duration $N$ is

\footnotetext{
${ }^{1}$ The principal has access to infinite penalty in our model and can implement the full-information allocation if auditing reveals the agent's income without any error.
} 
reached. The intuition for the periodicity is that the benefit of auditing is increasing with the duration of low-income reports, while the cost of auditing is constant. Auditing occurs only when the benefit exceeds the cost.

In our model, there are two instruments for providing incentives. One instrument is dynamic taxation that distorts the consumption path and makes future payoffs contingent on past history of reported incomes. This is the standard instrument used in dynamic mechanism design. For instance, Green (1987) uses this instrument to provide incentives for truthful reporting of income by designing taxes and subsidies that are history dependent. The second instrument is auditing: the principal has to pay a cost to use the instrument. The U.S. Internal Revenue Service uses the second instrument to provide incentives for taxpayers to pay their true share of taxes-those who are caught cheating are penalized. The principal in our model has access not only to the past history of reported incomes, but also to the history of auditing outcomes and, hence, can provide better incentives by using both instruments.

To understand the interaction between the two instruments, we study two versions of the model: one with the dynamic taxation instrument only and the other with both taxation and audit instruments. In the model with the dynamic taxation instrument only, to ensure that the high-income agent does not have the incentive to deviate, the low-income agent's consumption path is such that the static gain to the high-income agent from deviation is small, whereas the future losses are large. We show that this path is highly distorted since it implies a steeply declining consumption profile. We also show that when the sequence of low-income reports is sufficiently long, the distortion in the consumption path converges to infinity. In contrast, if the income process was independent and identically distributed (i.i.d.) as in Green (1987), the distortion is constant.

When the auditing instrument is also available, we show that the principal uses it to alleviate the distortion in the consumption path implied by the first instrument. Auditing reduces the distortion, because when the agent's income is observed during the audit, albeit imperfectly, the principal rewards the truth-teller relative to the cheater. In particular, the principal removes the distortion (accumulated up to the auditing date) in consumption for the truth-teller. This is not possible when there is no technology to ever verify who is the truth-teller and who is the cheater. We show that the optimal mechanism implies a discrete upward jump in the continuation utilities for the truthteller after the audit. We also show that no matter how high the cost of auditing is, there always exists a threshold instant at which the auditing probability becomes positive.

If the agent's absolute risk aversion is not constant, then he is audited minimally when risk aversion is either extremely high or extremely low. When risk aversion is extremely low, the variation in consumption incurs little cost; thus, there is no need to use the costly auditing instrument to reduce the distortion. When risk aversion is high, a small variation in consumption generates large incentive effects, hence there is again no need to use the auditing instrument. The model also implies that as the variance in income increases, auditing occurs more frequently.

In related literature on dynamic costly state verification, Wang (2005) studies deterministic auditing with i.i.d. hidden incomes. He finds that there is a critical level 
of auditing cost, below which there is auditing and above which there is no auditing. That is, if it is optimal to audit in one period, then it is optimal to audit in every period. Thus, the auditing in Wang (2005) is a static decision: it depends only on the cost of auditing and is independent of the past history. In our model, the auditing decision is dynamic and depends on past history via the duration of low-income reports. Popov (2007) studies stochastic auditing with i.i.d. hidden incomes. He specifies an exogenous lower bound for the agent's continuation utility and every cheater is moved to the lower bound if caught during the audit. In his model, auditing plays a dynamic role, but for agents with continuation utility at the lower bound, the auditing instrument is not effective because the principal cannot punish a liar any more. In our model, there is no lower bound on continuation utility and the auditing instrument is always effective. Monnet and Quintin (2005) study stochastic auditing with linear utility and i.i.d. hidden incomes. They find that auditing eventually is not used. We study the risk-averse case, thus consumption distortion plays a central role in our model.

Although we focus on tax compliance in the paper, the issue of fraud and optimal auditing is applicable to other areas in economics. For instance, a venture capitalist provides start-up funds to an entrepreneur to invent a new product. In the experimental stage, the entrepreneur receives outside funding, but after the product is invented, he might have to share the profits with the venture capitalist. If the outcome of the experiment is private information, then the entrepreneur can delay the report of being successful, keep the profit by selling the product privately, and continue to receive funding from the venture capitalist. In the problem of infant industry protection, domestic firms are subsidized for a certain period to help them increase their productivity and compete with foreign firms. If productivity is private information, the firms have strong incentives to cheat because they can earn monopoly rents and receive subsidies simultaneously. In the context of unemployment insurance, an unemployed worker might find a job at a random rate. The exact date when he finds the job might not be observable. By delaying the report of employment, the worker can receive both wage income and unemployment benefits.

The rest of the paper is organized as follows. Section 2 describes the basic model without auditing, and shows that the distortion in consumption increases with the duration of low-income reports. In Section 3, we introduce the auditing technology and show that it is optimal to audit the low-income agent periodically. Then we study the dependence of auditing frequency on the primitives of the model. Section 4 concludes. We provide the proofs of all the results in the Appendix.

\section{Model: No Auditing technology}

In this section we study a hidden income model in which the principal does not have access to an auditing technology. The characterization of the optimal contract in this section helps us examine the optimal auditing in Section 3 when an auditing technology is available. 
The tax authority is a risk-neutral principal with a discount rate $r>0$. The taxpayer is a risk-averse agent, whose preferences are given by

$$
E\left[\int_{0}^{\infty} r e^{-r t} u\left(c_{t}\right) d t\right]
$$

where $c_{t}$ is consumption at time $t, u(c)=-\exp (-\rho c)$ is a constant absolute risk-aversion (CARA) utility function with risk aversion $\rho, r$ is the discount rate (same as that of the principal), and $E$ is the expectation operator. Let $c:(-\infty, 0) \rightarrow \mathbb{R}$ denote the inverse of the utility function:

$$
c(u)=\frac{-\log (-u)}{\rho} .
$$

Agents have either high income, $w_{H}$, or low income, $w_{L}$, where $w_{L}<w_{H}$. All agents start with low income and transit to $w_{H}$ with Poisson rate $\pi>0$. For the model to be tractable, we assume that the high-income state is permanent. ${ }^{2}$

True income is not observable by the principal, so a high-income agent can underreport his income and pose as a low-income agent. We assume that the principal always asks the agent to show his reported income, so the low-income agent can never pretend to have $w_{H}$. Hence, there are no incentive constraints when the agent reports $w_{H}$.

The timing is as follows. In the initial period, the agent receives an income of either $w_{H}$ or $w_{L}$. He chooses to report either $w_{H}$ or $w_{L}$ to the principal. The principal assigns current and future consumptions based on the report. In subsequent periods, if an agent reported $w_{H}$ in the past, he is in an absorbing state and no further reports are necessary. If an agent reported $w_{L}$ in every period in the past, then he receives an income of either $w_{H}$ or $w_{L}$. The sequence of events then is the same as in the initial period.

The principal commits to delivering two sequences of consumptions, $\left\{\left(c^{H}(s)\right.\right.$, $\left.\left.c^{L}(s)\right) ; s \geq 0\right\}$, where $c^{H}(\cdot), c^{L}(\cdot):[0, \infty) \rightarrow \mathbb{R}$ are measurable functions. We denote this pre-commitment contract as $\sigma$. If an agent transits to $w_{H}$ at $t$, efficiency requires that the agent's consumption remain constant afterward. This is because the principal and the agent have the same discount rate and $w_{H}$ is an absorbing state. We denote this constant level of consumption as $c^{H}(t)$. The flow utility from this level of consumption then is $r u\left(c^{H}(t)\right)$. Let $H(t) \equiv u\left(c^{H}(t)\right)$ denote the discounted sum of utilities to an agent who transits to $w_{H}$ for the first time at $t$. Note that since true income is not observable, $H(t)$ is also the continuation utility to an agent who transited to $w_{H}$ before $t$, but reports $w_{H}$ for the first time at $t$. A low-income agent's consumption at $t$ is denoted by $c^{L}(t)$ and his flow utility is $u^{L}(t) \equiv u\left(c^{L}(t)\right)$. His continuation utility is

$$
\begin{aligned}
L(t) & \equiv \int_{t}^{\infty} e^{-(r+\pi)(\xi-t)}\left(\pi H(\xi)+r u^{L}(\xi)\right) d \xi \\
& =\int_{t}^{s} e^{-(r+\pi)(\xi-t)}\left(\pi H(\xi)+r u^{L}(\xi)\right) d \xi+e^{-(r+\pi)(s-t)} L(s), \quad \text { for all } t<s .
\end{aligned}
$$

\footnotetext{
${ }^{2}$ Similar simplifying assumptions are made by Hopenhayn and Nicolini (1997) in an unemployment insurance model and Golosov and Tsyvinski (2006) in a disability insurance model. We discuss in Section 3.4 how our results might be affected when the high-income state is not permanent.
} 
We refer to (1) as promise-keeping constraints.

The incentive compatibility constraint requires that an agent who transited to high income at $t$ does not have the incentive to delay the report of the transition to a later time $s$, i.e., to report $w_{L}$ from $t$ to $s$ and to report $w_{H}$ from $s$ onward:

$$
H(t) \geq \int_{t}^{s} e^{-r(\xi-t)} r u\left(c^{L}(\xi)+w_{H}-w_{L}\right) d \xi+e^{-r(s-t)} H(s) .
$$

The above constraint can be simplified as follows. CARA utility implies that $u\left(c^{L}(\xi)+\right.$ $\left.w_{H}-w_{L}\right)=u\left(c^{L}(\xi)\right)\left|u\left(w_{H}-w_{L}\right)\right|$. Define

$$
b \equiv\left|u\left(w_{H}-w_{L}\right)\right| \in(0,1),
$$

so the incentive constraint can be written as

$$
H(t) \geq \int_{t}^{s} e^{-r(\xi-t)} r b u^{L}(\xi) d \xi+e^{-r(s-t)} H(s) .
$$

The expected cost for the principal is

$$
C(\sigma)=\int_{0}^{\infty} e^{-(r+\pi) t}\left(\pi c^{H}(t)+r c^{L}(t)\right) d t .
$$

There should, in fact, be an additional term in $C(\sigma)$ : the discounted income obtained by the principal, $\left(r w_{L}+\pi w_{H}\right) /(r+\pi)$. However, unlike the unemployment insurance literature that endogenizes job-finding probabilities, the discounted income in our model is a constant, so it does not affect the optimal $\sigma$.

The principal's problem is to find an incentive compatible (I.C.) $\sigma$ that delivers a level of initial utility $L_{0}$ to a low-income agent and minimizes $C(\sigma)$, i.e.,

$$
\min _{\sigma} C(\sigma) \text { subject to } L_{0}=L(0) \equiv \int_{0}^{\infty} e^{-(r+\pi) t}\left(\pi H(t)+r u^{L}(t)\right) d t
$$

and constraint (2) for all $t<s$.

Next we obtain a recursive representation of the above problem. The promisekeeping constraint (1) and the incentive constraint (2) are equivalent to the following integral equations (4) and (5).

Lemma 1. The promise-keeping constraint (1) and the incentive constraint (2) hold for all $t<s$ if and only if

$$
\begin{aligned}
L(s)-L(t) & =\int_{t}^{s}\left((r+\pi) L(\xi)-\pi H(\xi)-r u^{L}(\xi)\right) d \xi \\
H(s)-H(t) & \leq \int_{t}^{s}\left(r H(\xi)-r b u^{L}(\xi)\right) d \xi
\end{aligned}
$$

hold for all $t<s$. 
Equation (4) states that the rate of change of $L$ is $r\left(L(t)-u^{L}(t)\right)+\pi(L(t)-H(t))$, where the first term is the rate of change of $L$ when there is no uncertainty (i.e., when there is no transition to the high-income state), and the second term captures the additional rate of change due to uncertainty. Inequality (5) plays the role of incentive constraints by imposing an upper bound on the rate of change of $H$. Intuitively, a sharply declining $H(\cdot)$ prevents the agent from delaying the high-income report. If (5) holds as an equality, then a high-income agent is indifferent between reporting $w_{H}$ at $t$ and delaying the high-income report until $s$; if (5) is strict, then delaying the high-income report makes him strictly worse off.

Following Fernandes and Phelan (2000) and Zhang (2009), we may write the principal's problem as a dynamic programming problem with $L$ and $H$ as the state variables, and with equations (4) and (5) as their laws of motion. With a slight abuse of notation, denote the principal's cost function as $C(L, H)$.

Remark 1. We include $H$ in the state variable for incentive reasons. The principal chooses $H(0)$ freely to minimize cost (i.e., $H(0) \in \arg \min _{H} C(L(0), H)$ ). In any continuation contract, however, $H$ is no longer a free variable, because $H$ acts as a threat utility. Raising $H(t)$ might induce an agent who transited to $w_{H}$ in earlier periods to postpone the high-income report until $t$.

Remark 2. The domain of the cost function $C(L, H)$ in the dynamic programming problem is $\{(L, H): L<H<0\}$. If $L$ is not strictly below $H$, then a high-income agent would pose as a low-income agent and consume more than the low-income agent.

In the rest of this section, we study the optimal contract with no auditing in three steps. In Section 2.1, we show a homogeneity property of the cost function $C(L, H)$ and use it to introduce a measure of consumption distortion. In Section 2.2, we use the homogeneity property to obtain a simplified Hamilton-Jacobi-Bellman equation. Then we fully characterize the optimal contract by showing that the cost function is the unique solution to this Hamilton-Jacobi-Bellman equation. In Section 2.3, we explain why the distortion increases with the duration of low-income reports, which helps us understand the optimal auditing in Section 3.

\subsection{A measure of distortion}

Recall that the agent's utility function belongs to the CARA class. A property of the utility function is that

$$
-\exp \left(-\rho\left(c-\frac{\log (\alpha)}{\rho}\right)\right)=-\alpha \exp (-\rho c) \quad \text { for all } \alpha>0
$$

Suppose that a contract $\sigma \equiv\left\{\left(c^{L}(t), c^{H}(t)\right) ; t \geq 0\right\}$ delivers the continuation utility pair $(L, H)$. Then a contract

$$
\sigma_{\alpha} \equiv\left\{\left(c^{L}(t)-\frac{\log (\alpha)}{\rho}, c^{H}(t)-\frac{\log (\alpha)}{\rho}\right) ; t \geq 0\right\}
$$


delivers $(\alpha L, \alpha H)$. The reverse is also true. Further, $\sigma$ is I.C. if and only if $\sigma_{\alpha}$ is I.C. Therefore, $\left\{\left(c^{L *}(t), c^{H *}(t)\right) ; t \geq 0\right\}$ is the optimal contract to deliver $(L, H)$ if and only if $\left\{\left(c^{L *}(t)-\log (\alpha) / \rho, c^{H *}(t)-\log (\alpha) / \rho\right) ; t \geq 0\right\}$ is the optimal contract to deliver $(\alpha L, \alpha H)$. The next lemma states this homogeneity property and is used to obtain a measure of distortion.

LEMma 2. The cost function $C$ has the following properties.

(i) Homogeneity: For any $\alpha>0$,

$$
C(\alpha L, \alpha H)=C(L, H)-\frac{\log (\alpha)}{\rho} .
$$

(ii) Monotonicity: The cost $C(L, H)$ is weakly decreasing in $H$.

Recall that $c(\cdot)$ is the inverse of the utility function, so (6) is the same as

$$
C(\alpha L, \alpha H)=C(L, H)+c(-\alpha) .
$$

We can thus decompose the cost $C(L, H)$ as

$$
C(L, H)=C\left(|L|(-1),|L|\left(-\frac{H}{L}\right)\right)=C\left(-1,-\frac{H}{L}\right)+c(L) .
$$

With full information and no incentive constraints, the principal delivers $L$ to the lowincome agent via a stream of constant consumption, i.e., $H(\cdot)=u^{L}(\cdot)=L$ in problem (3). Hence the cost of delivering $L$ under full information is $c(L)$. The distortion of consumption to the low-income agent in our contract can be measured by the difference between the cost $C(L, H)$ and the full-information cost $c(L)$ :

$$
C(L, H)-c(L)=C\left(-1,-\frac{H}{L}\right) \equiv \hat{c}(x)
$$

where

$$
x \equiv \frac{H}{L} \quad \text { and } \quad \hat{c}(x) \equiv C(-1,-x) .
$$

It is helpful to compare the distortion in our model to that in Green (1987). With i.i.d. incomes, private information, and CARA utility, Green (1987) shows that the cost function implied by the optimal contract differs from the full-information cost function only by a constant. Thus, the distortion in any continuation contract in the i.i.d. case is constant. In particular, the distortion is independent of the history or the level of evolving continuation utilities. With persistent shocks, the distortion is independent of the level of the continuation utility $L$, but depends on the ratio $H / L$, as noted in (7). Part (ii) of Lemma 2 implies that the higher is the ratio (i.e., lower $H$ ), the higher is the distortion. 


\subsection{The optimal contract without auditing}

In this section, we first derive a Hamilton-Jacobi-Bellman (HJB) equation for the cost function. Then we conjecture a candidate solution to the HJB equation. Finally, we verify that the allocation implied by the candidate solution solves problem (3). The last step of verifying the optimality of the candidate solution is needed, because the HJB equation has multiple solutions.

If $L(\cdot)$ and $H(\cdot)$ are differentiable, we can rewrite (4) and (5) as differential equations and inequalities:

$$
\begin{aligned}
\frac{d L(t)}{d t} & =(r+\pi) L(t)-\pi H(t)-r u^{L}(t) \\
\frac{d H(t)}{d t} & \leq r H(t)-r b u^{L}(t) .
\end{aligned}
$$

Note that we allow for downward jumps in $H(\cdot)$ : when $H(t)>\lim _{s \downarrow} H(s)$, we interpret the discontinuity as $d H(t) / d t=-\infty$, and the differential inequality (8) still holds under this interpretation. Introducing a slack variable $\mu(t) \geq 0$ in (8), we get

$$
\begin{aligned}
& \min _{\sigma} C(\sigma)=\int_{0}^{\infty} e^{-(r+\pi) t}\left(\pi c(H(t))+r c\left(u^{L}(t)\right)\right) d t \\
& \text { subject to } \frac{d L(t)}{d t}=(r+\pi) L(t)-\pi H(t)-r u^{L}(t) \\
& \frac{d H(t)}{d t}=r H(t)-r b u^{L}(t)-\mu(t) .
\end{aligned}
$$

The HJB equation for the cost function $C(L, H)$ is

$$
(r+\pi) C(L, H)=\min _{u^{L}, \mu}\left\{C_{L}(L, H) \frac{d L}{d t}+C_{H}(L, H) \frac{d H}{d t}+r c\left(u^{L}\right)+\pi c(H)\right\} .
$$

We can simplify (12) by replacing the state $(L, H)$ with $(L, x)$. Equations (10) and (11) imply that

$$
\frac{d x}{d t}=\frac{d\left(\frac{H}{L}\right)}{d t}=r(x-b) \frac{u^{L}}{L}+\pi(x-1) x-\frac{\mu}{L} .
$$

Recall that the homogeneity property allows us to write $C(L, H)=c(L)+\hat{c}(x)$. The HJB equation (12) can be written as

$$
(r+\pi)(c(L)+\hat{c}(x))=\min _{u^{L}, \mu}\left\{c^{\prime}(L) \frac{d L}{d t}+\hat{c}^{\prime}(x) \frac{d x}{d t}+r c\left(u^{L}\right)+\pi c(H)\right\},
$$

which, after rearrangement, becomes

$$
\begin{aligned}
(r+\pi) \hat{c}(x)=\min _{u^{L}, \mu}\{ & -\frac{1}{\rho} \frac{(r+\pi) L-\pi H-r u^{L}}{L} \\
& \left.+\hat{c}^{\prime}(x)\left(r(x-b) \frac{u^{L}}{L}+\pi(x-1) x-\frac{\mu}{L}\right)+r c\left(-\frac{u^{L}}{L}\right)+\pi c\left(-\frac{H}{L}\right)\right\} .
\end{aligned}
$$


Introducing $\hat{u}^{L} \equiv u^{L} / L>0$ and $\hat{\mu} \equiv-\mu / L \geq 0$, we get

$$
\begin{aligned}
(r+\pi) \hat{c}(x)=\min _{\hat{u}^{L}, \hat{\mu}}\{- & \frac{1}{\rho}\left(r+\pi-\pi x-r \hat{u}^{L}\right) \\
& \left.+\hat{c}^{\prime}(x)\left(r(x-b) \hat{u}^{L}+\pi(x-1) x+\hat{\mu}\right)+r c\left(-\hat{u}^{L}\right)+\pi c(-x)\right\} .
\end{aligned}
$$

Note that the homogeneity property makes the state space one dimensional, even though the state space in (9) contains two dimensions.

Next we make a conjecture to narrow down candidate solutions to (14): there exists an $x^{*} \in(0,1)$, to the left of which $\hat{c}(\cdot)$ is flat and to the right of which it is strictly increasing. ${ }^{3}$ If we know the optimal contract starting with $x(0)=x^{*}$, then the optimal contract with $x(0)<x^{*}$ can be obtained by a discrete jump in the state variable to $x^{*}$. Therefore, we focus on the solution to (14) in $\left[x^{*}, 1\right)$. Because $\hat{c}(\cdot)$ is strictly increasing in $\left[x^{*}, 1\right)$, it is optimal to choose $\hat{\mu}=0$. Hence we can remove $\hat{\mu}$ from the right side of (14) and restrict attention to solutions that are monotonically increasing in $x$. That is, we rewrite (14) as

$$
\hat{c}(x)=\mathscr{H}\left(\hat{c}^{\prime}(x), x\right) \quad \text { and } \quad \hat{c}^{\prime}(x) \geq 0,
$$

where

$$
\begin{aligned}
\mathscr{H}(d, x) \equiv \frac{1}{r+\pi} \min _{\hat{u}^{L}}\left\{-\frac{1}{\rho}(r+\pi\right. & \left.-\pi x-r \hat{u}^{L}\right) \\
& \left.+d\left(r(x-b) \hat{u}^{L}+\pi(x-1) x\right)+r c\left(-\hat{u}^{L}\right)+\pi c(-x)\right\} .
\end{aligned}
$$

In the following, we solve (15) as an initial value problem by expressing $\hat{c}^{\prime}$ as a function of $x$ and $\hat{c}$. We establish the domain for $(x, \hat{c})$ by showing some properties of $\mathscr{H}$ in the lemma below.

Lemma 3. (i) The function $\mathscr{H}(0, x)$ decreases in $x$ (see Figure 1).

(ii) Denote the maximizer in $\max _{d \geq 0} \mathscr{H}(d, x)$ as $\bar{d}(x)$. Then

$$
\bar{d}(x) \begin{cases}=0 & \text { if } x \leq \underline{x} \\ >0 & \text { if } x>\underline{x}\end{cases}
$$

where $\underline{x}$ is the unique root in $(b, 1)$ to the equation $r(x-b)+\pi(x-1) x=0 .{ }^{4}$

(iii) If $x>\underline{x}$, then $\mathscr{H}(\cdot, x)$ is strictly increasing in $(0, \bar{d}(x))$.

\footnotetext{
${ }^{3}$ Because the principal minimizes the cost, it is reasonable to conjecture that $\hat{c}(\cdot)$ is a convex function. Let $x^{*}$ be the largest minimizer in $\arg \min _{x} \hat{c}(x)$. Then $\hat{c}(\cdot)$ is flat on $\left(0, x^{*}\right]$ for two reasons: (i) $\hat{c}(x) \geq \hat{c}\left(x^{*}\right)$ $\forall x \in\left(0, x^{*}\right]$ because $x^{*}$ is a minimizer; (ii) $\hat{c}(x) \leq \hat{c}\left(x^{*}\right) \forall x \in\left(0, x^{*}\right]$ because $\hat{c}(\cdot)$ is nondecreasing. It is easy to see that $\hat{c}(\cdot)$ is strictly increasing on $\left[x^{*}, 1\right)$. Convexity implies that $\hat{c}^{\prime}(x)$ is nondecreasing in $x$. If $\hat{c}^{\prime}(y)=0$ for some $y>x^{*}$, then $\hat{c}^{\prime}(x)=0 \forall x \in\left[x^{*}, y\right]$, contradicting the fact that $x^{*}$ is the largest minimizer.

${ }^{4}$ The quadratic function $f(x) \equiv r(x-b)+\pi(x-1) x$ has a unique root in $(b, 1)$ because $f(b)<0$ and $f(1)>0$. So one root is between $b$ and 1 , and the other is below $b$.
} 


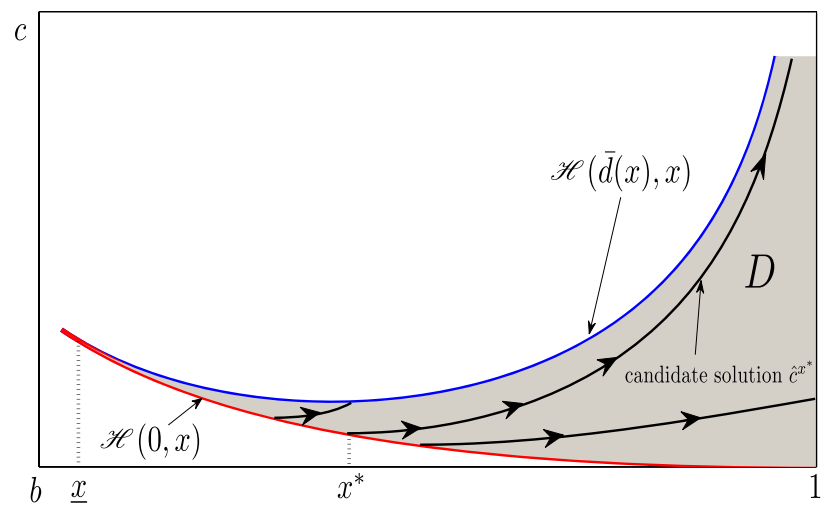

FIGURE 1. The candidate solution with no auditing.

Part (ii) in Lemma 3 shows that $\mathscr{H}(0, x)<\mathscr{H}(\bar{d}(x), x)$ if and only if $\underline{x}<x$. Define $D$ as the region between $\mathscr{H}(0, x)$ and $\mathscr{H}(\bar{d}(x), x)$,

$$
D \equiv\{(x, \hat{c}): \underline{x}<x<1, \mathscr{H}(0, x)<\hat{c}<\mathscr{H}(\bar{d}(x), x)\} .
$$

For any $(x, \hat{c}) \in D$, let $d^{*}(x, \hat{c})$ be the inverse of $\mathcal{H}$, that is, the root of $\mathscr{H}(d, x)=\hat{c}$. It follows from part (iii) in Lemma 3 that this root exists and is unique in $(0, \bar{d}(x))$. Thus, the HJB equation (15) is equivalent to the following initial value problem in $D$ :

$$
\hat{c}^{\prime}(x)=d^{*}(x, \hat{c}(x)) \text {. }
$$

To solve (16), we start with the initial condition, $\left(x^{*}, \mathcal{H}\left(0, x^{*}\right)\right)$, since $\hat{c}^{\prime}\left(x^{*}\right)=0$ and $\hat{c}\left(x^{*}\right)=\mathscr{H}\left(0, x^{*}\right)$. Then the solution curve enters $D$ (see Figure 1) because $x^{*}>\underline{x} .{ }^{5}$ Because $x^{*}$ is unknown a priori, we find a condition to pin down $x^{*}$ in the next subsection.

2.2.1 Candidate solution Let $\hat{c}^{x_{0}}(\cdot)$ denote the solution to (16) with initial condition $\left(x_{0}, \mathscr{H}\left(0, x_{0}\right)\right)$. Figure 1 shows the solutions for various $x_{0}$. These solutions are ordered by their function values: a solution starting with a lower initial value $\hat{c}^{x_{0}}\left(x_{0}\right)=\mathscr{H}\left(0, x_{0}\right)$ remains low as long as it resides in $D$. (Note that lower function value occurs with higher initial state $x_{0}$.) There is a unique $x^{*}$ such that the solution $\hat{c}^{x^{*}}(\cdot)$ stays in $D$ forever. We call $\hat{c}^{x^{*}}(\cdot)$ the candidate solution. In Section 2.2.2, we verify that the allocation implied by the candidate solution is indeed optimal. If a solution is lower than $\hat{c}^{x^{*}}(\cdot)$, then the solution curve reaches the upper limit of the domain at 1 . This is inappropriate because no contract can deliver equal continuation utilities to both types (i.e., 1 cannot be in the domain of $\hat{c}(\cdot)$ ). If a solution is higher than $\hat{c}^{x^{*}}(\cdot)$, then it rises too rapidly and crosses the upper boundary $\mathscr{H}(\bar{d}(\cdot), \cdot)$, which is also inappropriate as $\hat{c}(x) \leq \max _{d \geq 0} \mathscr{H}(d, x)$. We prove these results in the following lemma.

\footnotetext{
${ }^{5}$ It is easy to see that $x^{*}$ exceeds $\underline{x}$; otherwise, since $\hat{c}(x)$ increases and $\mathscr{H}(\bar{d}(x), x)$ decreases in $x$ (because it coincides with $\mathscr{H}(0, x)$ when $x \leq \underline{x}$; see parts (i) and (ii) in Lemma 3$)$, the function $\hat{c}(x)$ exceeds $\mathscr{H}(\bar{d}(x), x)$ immediately after $x^{*}$, which violates the HJB equation.
} 
Lemma 4. (i) For each initial condition $\left(x_{0}, \mathcal{H}\left(0, x_{0}\right)\right)$ with $x_{0} \in(\underline{x}, 1)$, the solution $\hat{c}^{x_{0}}(\cdot)$ to (16) exists and is unique in D. Further, it is strictly increasing and strictly convex when $x \geq x_{0}$. The cost function $C(L, H)=c(L)+\hat{c}^{x_{0}}(H / L)$ is strictly convex when $L<0$ and $H / L \geq x_{0}$. The optimal policy $\hat{u}^{L}$ in $\mathcal{H}\left(\left(\hat{c}^{x_{0}}\right)^{\prime}(x), x\right)$ implies $d x / d t>0$ (i.e., distortion increases with the duration of low-income reports).

(ii) There is a unique initial condition, $\left(x^{*}, \mathcal{H}\left(0, x^{*}\right)\right)$, such that the solution $\hat{c}^{x^{*}}(\cdot)$ does not exit $D$.

When $x<x^{*}$, the state takes a discrete jump to $x^{*}$. Hence we extend $\hat{c}^{x^{*}}(\cdot)$ to $\left(0, x^{*}\right)$ by $\hat{c}^{x^{*}}(x) \equiv \hat{c}^{x^{*}}\left(x^{*}\right)$ for $x<x^{*}$. The candidate solution $\hat{c}^{x^{*}}(\cdot)$ satisfies the HJB equation (14) in $\left[x^{*}, 1\right)$ by definition. However, (14) is violated when $x<x^{*}$, because $\left(\hat{c}^{x^{*}}\right)^{\prime}(x)=0$ implies

$$
\hat{c}^{x^{*}}(x)=\hat{c}^{x^{*}}\left(x^{*}\right)<\frac{1}{r+\pi} \min _{\hat{u}^{L}, \hat{\mu}}\left\{-\frac{1}{\rho}\left(r+\pi-\pi x-r \hat{u}^{L}\right)+r c\left(-\hat{u}^{L}\right)+\pi c(-x)\right\} .
$$

This reinforces the intuition that the state variable $x$ needs to exit $\left(0, x^{*}\right)$ immediately.

The candidate solution $\hat{c}^{x^{*}}(\cdot)$ implies an allocation. For each $x \geq x^{*}$, the optimal policy $\hat{u}^{L}$ is determined as $1 /\left(1+\rho(x-b)\left(\hat{c}^{x^{*}}\right)^{\prime}(x)\right)$, and the state variables evolve as in (10), (11), and (13). In the next subsection, we verify that this implied allocation is optimal.

2.2.2 Verification Given the initial promised utilities $(L, H)$, the following lemma shows that

(i) the cost of the contract implied by $\hat{c}^{x^{*}}(\cdot)$ is $C(L, H)$

(ii) the costs of other I.C. contracts are weakly higher than $C(L, H)$.

The main technique behind the proof is to integrate the HJB equation from time 0 to time $T$ and then take limit $T \rightarrow \infty$.

Lemma 5. When the auditing technology is not available, the contract implied by $\hat{c}^{x^{*}}(\cdot)$ is optimal.

\subsection{Discussion}

We explain in this subsection why the distortion trends upward. Our explanation has two parts. First, we provide some intuition for why the distortion is an increasing function of the ratio $H / L$. Second, we show that $H / L$ increases with the duration of lowincome reports. Under persistent information, the principal lets $H$ fall faster than $L$ conditional on the low-income report, while in the i.i.d. case, they both fall at the same rate.

2.3.1 Why does higher $x$ imply higher distortion? We have seen from Figure 1 that the distortion $\hat{c}^{x^{*}}$ approaches infinity as $x \rightarrow 1$. This result might be puzzling, as the continuation utility $H$ approaches $L$ when $x \rightarrow 1$, which is exactly the feature of the fullinformation contract. To resolve this puzzle, let us compare the consumption paths 
when $H$ approaches $L$, with and without private information. With full information, consumption of a high-income agent approaches that of a low-income agent whenever $H$ approaches $L$, because the mapping between continuation utility and consumption is one-for-one. This one-for-one relationship no longer holds with private information. The high-income agent's consumption remains un-distorted with private information. The low-income agent's consumption path, however, is very distorted when $H$ is just slightly above $L$ due to the incentive constraints. To see this, note that $\lim _{x \rightarrow 1}\left(\hat{c}^{x^{*}}\right)^{\prime}(x) \rightarrow \infty$. When $H$ is close to $L$, the low-income agent's flow utility $u^{L}=\left(1 /\left(1+\rho(x-b)\left(\hat{c}^{x^{*}}\right)^{\prime}(x)\right)\right) L$ is close to zero. The large consumption at that instant needs to be offset by much lower levels of consumption in the future, so as to deliver a given level of continuation utility $L$ (to confirm this intuition, we show in Lemma 10 that both $L(t)$ and $H(t)$ decrease with time) ${ }^{6}$ The closer $H$ is to $L$, the more a low-income agent's consumption is front-loaded and, hence, the faster his continuation utility declines. Thus, higher $x$ implies higher distortion.

2.3.2 Why does $x$ increase with the duration of low-income reports? To see the intuition for why $H / L$ increases with the duration of low-income reports, we start with a threeperiod model in which the agent has random income, either $w_{H}$ or $w_{L}$, in the first two periods. There is no discounting. In the last period, the agent has no income, hence there is no need for income reports (and there are no incentive problems). We keep the last period in the model so that the principal can provide incentives in the second period when income is private. Income in the first period is private, and income in the second period is public in Case 1 and private in Case 2. Here we focus on an agent who has low income in the first period. The allocation for an agent who has high income in the first period is not discussed, as it is irrelevant.

Case 1. Income is private in the first period, but public in the second. Suppose that income is i.i.d. The low-income agent's continuation utility at the beginning of the second period is

$$
L(2)=\pi_{L} V_{L}+\pi_{H} V_{H},
$$

where $\pi_{i} \equiv \operatorname{Pr}\left(w_{i}\right)$ and $V_{i} \equiv u\left(c_{i}(2)\right)+u\left(c_{i}(3)\right)$ is the utility conditional on the second period income $w_{i}, i=L, H$. Because income is i.i.d., the low-income agent's continuation utility, $L(2)$, is also the liar's continuation utility. It must be made lower than that for a high-income agent, which is the dynamic reward versus punishment aspect of dynamic contracts studied in Green (1987). Because income in the second period is observable, the principal offers full insurance starting from the second period and chooses $V_{H}=V_{L}$. When income is persistent, this equality fails, as the informational content of observing $w_{H}$ in the second period is different from observing $w_{L}: w_{H}$ in the second period is more suggestive of a liar than $w_{L}$, hence, the principal finds it optimal to choose $V_{H}<V_{L}$. This is akin to the moral-hazard (hidden-effort) model, where the agent's consumption is lower in states that are more likely to be realized with shirking. In the following discussion, we offer an alternative interpretation related to the above information-content

\footnotetext{
${ }^{6}$ Here the low-income agent is savings constrained. In dynamic risk-sharing problems with private information, optimal contracts typically restrict agents' savings (Rogerson 1985, Green 1987).
} 
intuition, but more useful for our purpose: in the persistent case, what matters for the incentive is not only $L(2)$, but also the difference between $V_{H}$ and $V_{L}$. Because a liar is more likely to receive $V_{H}$ (denote the liar's probability of receiving $w_{H}$ in the second period as $\tilde{\pi}_{H}, \tilde{\pi}_{H}>\pi_{H}$ ), his continuation utility is

$$
L(2)+\left(\tilde{\pi}_{H}-\pi_{H}\right)\left(V_{H}-V_{L}\right) .
$$

The larger the difference between $V_{H}$ and $V_{L}$, the more a liar could gain from the continuation contract for the low-income agent. With persistence, the principal has access to a second instrument to reduce the liar's gain: keep $L$ (2) fixed but lower $V_{H}-V_{L}$. This instrument is ineffective in the i.i.d. case, as the liar cares only about $L(2)$. With persistence, this instrument is always used, as a small deviation from $V_{H}=V_{L}$ has a first-order effect on the incentive constraint, but incurs only a second-order cost. In fact, here the principal makes $V_{H}-V_{L}$ negative.

Case 2. Income is private in both the first and the second periods. Different from Case 1, here $V_{H}$ must be above $V_{L}$; otherwise, the agent does not reveal his secondperiod income truthfully. However, the key insight from the previous case remains. With i.i.d. incomes, only the continuation utility (the probability weighted average of $V_{H}$ and $V_{L}$ ) matters for the incentives; with persistent incomes, both the continuation utility and the difference $V_{H}-V_{L}$ matter. With i.i.d. incomes, the agent's continuation utility falls with the first period's low-income report; with persistent incomes, the average of $V_{H}$ and $V_{L}$ still falls, but the principal lets $V_{H}$ fall faster than $V_{L}$ to further reduce a liar's gain.

The intuition from the three-period model carries over to an infinite-horizon model. Consider an infinite-horizon discrete-time model with i.i.d. income. Each period has a length $d t$, and the income is $w_{H}$ with probability $\pi d t$ and is $w_{L}$ with probability $1-$ $\pi d t$. Green (1987) shows that this problem has only one state variable $L(t)$, which is the agent's continuation utility before the realization of income in period $t$. Recall that $H(t)$ denotes the agent's continuation utility after the realization of high income in period $t$. The Bellman equation is

$$
\begin{aligned}
C(L(t))=\min _{H(t), c^{L}(t)}\left\{\pi d t \mathbf{C}(H(t))+(1-\pi d t)\left[r d t c^{L}(t)+(1-r d t) C(L(t+d t))\right]\right\} \\
\text { subject to } H(t) \geq(r d t) b u^{L}(t)+(1-r d t) L(t+d t) \\
L(t)=\pi d t H(t)+(1-\pi d t)\left[(r d t) b u^{L}(t)+(1-r d t) L(t+d t)\right],
\end{aligned}
$$

where $\mathbf{C}(H(t))$ is the cost following a high-income report. (We do not state the problem following a high-income report explicitly because we want to focus on a path with only low-income reports.) With CARA preferences, Green (1987) shows two properties of this problem: (i) $C(L(t))$ and $\mathbf{C}(H(t))$ differ from the full-information cost function only by a constant; (ii) although continuation utility $L(t)$ falls with the duration of lowincome reports (i.e., $L(t)>L(t+d t)$ ), the ratio $x(t)=H(t) / L(t)$ is constant. That $x(t)$ remains constant is because of the homogeneity property: policy functions (including current and future utilities) in a continuation contract are all proportional to $L(t)$. With i.i.d. incomes, $H(t)$ is a control variable and is optimized every period given $L(t)$; in contrast, in our model with persistent incomes, $H(t)$ is also a state variable. The differ- 
ence $H(t)-L(t)$ is the counterpart of the difference $V_{H}-V_{L}$ in the above three-period example. The Bellman equation when the high-income state is permanent is

$$
\begin{aligned}
C(L(t), H(t))=\min _{c^{L}(t)}\{\pi d t & \underline{c(H(t))} \\
\left.+(1-\pi d t)\left[r d t c^{L}(t)+(1-r d t) C(L(t+d t), H(t+d t))\right]\right\} & \\
\text { subject to } H(t) & \geq(r d t) b u^{L}(t)+(1-r d t) \underline{H(t+d t)} \\
L(t) & =\pi d t H(t)+(1-\pi d t)\left[(r d t) b u^{L}(t)+(1-r d t) L(t+d t)\right] .
\end{aligned}
$$

The two underlined terms reflect the differences from the i.i.d. case. The first difference is immaterial because $c(H(t))$ and $\mathbf{C}(H(t))$ differ only by a constant. The second difference (i.e., $H(t+d t)$ replaces $L(t+d t)$ in the right side of the incentive constraint) accelerates the speed at which $H(t+d t)$ falls, because smaller $H(t+d t)$ makes it easier to satisfy the incentive constraint. Recall that $H(t)$ is proportional to $L(t)$ in the i.i.d. case. In contrast, $H(t)$ falls faster than $L(t)$ in our model. As a result, $x=H / L$ increases with the duration of low-income reports.

\section{Model: Costly ANd Imperfect Auditing}

When an auditing technology is available, the principal has access to two instruments. In addition to distorting the consumption path to provide incentives, now the principal can deter cheating by auditing the agent's report. Since high income is an absorbing state, it is easy to see that auditing is unnecessary forever if the agent reports $w_{H}$ just once in the past. Auditing costs $\gamma$ units of consumption good. However, auditing is not perfect: there is a positive probability $\varpi>0$ of auditing error that labels a lowincome agent as having high income. If a low-income agent is audited after reporting $w_{L}$, the principal observes either a low-income signal $\mathcal{L}$ with probability $1-\varpi$ or a highincome signal $\mathcal{H}$ with probability $\varpi$. However, there is no auditing error that labels a high-income agent as having low income, i.e., if a high-income agent is audited after reporting $w_{L}$, the principal observes $\mathcal{H}$ with probability 1 .

The timing is as follows. In the initial period $(t=0)$, the agent receives an income of either $w_{H}$ or $w_{L}$. He chooses to report either $w_{H}$ or $w_{L}$ to the principal. Then conditional on the low-income report, the principal chooses to audit according to a Poisson arrival rate $p(0)$. (Recall that auditing is unnecessary if the report is $w_{H}$.) The principal assigns current and future consumptions, conditional on the report, whether the audit occurs, and the signal $\mathcal{L}$ or $\mathcal{H}$ if the audit does occur. In subsequent periods, if an agent reported $w_{H}$ in the past, he is in an absorbing state and no further reports or auditing are necessary. If an agent reported $w_{L}$ in every period in the past, then he receives an income of either $w_{H}$ or $w_{L}$. The sequence of events then is the same as in the initial period.

Note that since our model is in continuous time, $p(t)$ is the endogenous arrival rate of an audit at instant $t$, not the auditing probability itself. That is, over a period of length $d t$, the principal audits with probability $p(t) d t$ and does not audit with probability $1-p(t) d t$. If $p(t)=0$, no audit arrives, while if $p(t)=\infty$, the auditing probability has an atom at $t$. 
Conditional on signals from the audit, the principal assigns new continuation utilities to the agents. Let $L_{\mathcal{L}}(t)$ and $L_{\mathcal{H}}(t)$ be the continuation utilities of an audited lowincome agent with signals $\mathcal{L}$ and $\mathcal{H}$, respectively, at $t$. Let $H_{\mathcal{H}}(t)$ be the continuation utility of an audited high-income agent (whose signal can only be $\mathcal{H}$ ) at $t$. Finally, let $H_{\mathcal{L}}(t)$ be the continuation utility of an audited low-income agent with signal $\mathcal{L}$ who transited to high income immediately after he was audited.

The principal pre-commits not only to the two sequences of consumption, as in the previous section, but also to the sequence of arrival rates of audit. We can again represent the principal's cost minimization problem recursively with $L$ and $H$ as state variables. The cost must be weakly less than that in Section 2, since it is always feasible for the principal to shut down auditing. We continue to exclude the discounted income from the cost function $C(L, H)$ for the same reason as in Section 2; however, $C(L, H)$ now includes both the cost of delivering consumption and the cost of auditing.

When the principal audits, (i) it is feasible for her to deliver any continuation utility pair $(L, H)$, such that $L<0$ and $H<0$, and (ii) $\lim _{H \downarrow-\infty} C(L, H)<\infty$ (this contrasts with $\lim _{H \downarrow L} C(L, H)=\infty$ in Section 2 and is explained below in Remark 3). These statements imply that the full-information constant consumption can be achieved. To see this, suppose that the principal wants to implement $(L, L)$ and offers constant consumption $c(L)$ to the agent regardless of income reports. This is, however, not incentive compatible, as the high-income agent wants to underreport. To deter cheating, the principal randomly jumps from state $(L, L)$ to state $(L, H)$ at a small rate $\epsilon>0$ conditional on the low-income report. For any $\epsilon>0, H$ can be made sufficiently low so as to restore the incentive compatibility of the contract. Because each jump incurs a finite cost (because $C(L, \cdot)$ is bounded), the total cost incurred by the jumps diminishes when $\epsilon$ is made arbitrarily small.

Remark 3. It is easy to see that $\lim _{H \downarrow-\infty} C(L, H)<\infty$ when auditing is perfect. With no auditing error, the principal could perfectly separate the two types of agents after paying a finite cost $\gamma$. If the agent's income is truly low, the principal could reset $H$ to the optimal level without affecting incentives, hence $C(L, H) \leq \gamma+\min _{\tilde{H}} C(L, \tilde{H})$ for all $H$. With auditing error, the principal could still partially separate the two types of agents by auditing multiple times in a small time interval, say in $[0, \epsilon]$. Consider a contract starting at state $(L, H)$, where $H$ is low: $c^{H}(t)=c(H)$ and $c^{L}(t)=c(H)-\left(w_{H}-w_{L}\right) \forall t \geq 0$. In this contract, the high-income agent is indifferent between reporting $w_{H}$ and $w_{L}$. Hence, this contract is incentive compatible and no auditing is needed for incentive purposes. However, the low-income agent's promise-keeping constraint is violated when $H<L$. To solve this problem, the principal uses auditing to partially discover the low-income agent and reward him. She audits at instants $(j / J) \epsilon, j=1,2, \ldots, J$, and stops when the signal $\mathcal{L}$ arrives for the first time. Denote the first arrival time as $\tau$ (if $\mathcal{L}$ has not arrived until $\epsilon$, let $\tau=\infty$ ). Since the principal observes only signal $\mathcal{H}$ from the high-income agent and observes signal $\mathcal{L}$ from the low-income agent sooner or later, this auditing strategy discovers the low-income agent when $J$ is large. For the principal to observe a sequence of $\mathcal{H}$ signals of length $J$ from the low-income agent, the agent either must be extremely unlucky or must have transited to high income before $\epsilon$. If $\epsilon$ is small, the 
auditing cost is finite even when $J$ is large. ${ }^{7}$ At $\tau$, the principal resets $L(\tau)=L^{*}$ to satisfy the promise-keeping constraint. If $\epsilon$ is small and $J$ is large, $L^{*}$ must be close to $L$, as the low-income agent's probability of staying in the above contract is very small. The principal also resets $H(\tau)$ to the optimal level, i.e., $H(\tau) \in \arg \min _{\tilde{H}} C\left(L^{*}, \tilde{H}\right)$, without violating the incentive constraint, as a high-income agent cannot generate a signal $\mathcal{L}$ and, hence, he does not benefit from the resetting. To summarize, the principal can reduce the cost $C(L, H)$ to $\min _{\tilde{H}} C\left(L^{*}, \tilde{H}\right)$ by incurring a finite auditing cost in a small time interval. Hence $C(L, H)$ is bounded above no matter how low $H$ is.

The previous discussion and Remark 3 imply that without further restrictions on the domain of the cost function, the principal can deliver the full-information constant consumption. To make the problem nontrivial, we exclude the region $\{(L, H): H<L<0\}$ from the domain of the cost function. If $H<L<0$, the agent is induced not to transit to the high-income state: he can secretly decline the $w_{H}$ offer and continue to stay in the low-income state.

In the rest of this section, the domain of the cost function is restricted to $\{(L, H)$ : $L \leq H<0\}$. To see that the principal can deliver any $(L, H)$ in the restricted domain, recall from Section 2 that she can deliver $L<H<0$, even when the auditing technology is not available. Auditing helps the principal deliver $L=H<0$, as shown in Remark 4 below.

For now we impose a restriction that atomic auditing is not allowed. In Section 3.2, we verify that the principal does not use atomic auditing even if it is allowed. When there is no atomic auditing (i.e., $p(\cdot)<\infty$ ), the probability that auditing does not occur in $[t, s)$, conditional on the history up to time $t$, is $e^{-\int_{t}^{s} p(\xi) d \xi}$. The promise-keeping constraint is

$$
\begin{aligned}
L(t)=\int_{t}^{s} e^{-(r+\pi)(\xi-t)-\int_{t}^{\xi} p(\eta) d \eta}\left(r u^{L}(\xi)\right. & \left.+\pi H(\xi)+p(\xi)\left((1-\varpi) L_{\mathcal{L}}(\xi)+\varpi L_{\mathcal{H}}(\xi)\right)\right) d \xi \\
& +e^{-(r+\pi)(s-t)-\int_{t}^{s} p(\xi) d \xi} L(s) \quad \text { for all } t<s .
\end{aligned}
$$

The incentive constraint is

$$
\begin{aligned}
H(t) \geq \int_{t}^{s} e^{-r(\xi-t)-\int_{t}^{\xi} p(\eta) d \eta}\left(r b u^{L}(\xi)+p(\xi) H_{\mathcal{H}}(\xi)\right) d \xi & \\
& +e^{-r(s-t)-\int_{t}^{s} p(\xi) d \xi} H(s) \text { for all } t<s .
\end{aligned}
$$

Using the same arguments as in Lemma 1, we know that the promise-keeping constraint (17) and the incentive constraint (18) hold for all $t<s$ if and only if

$$
\begin{gathered}
L(s)-L(t)=\int_{t}^{s}\left((r+\pi+p(\xi)) L(\xi)-r u^{L}(\xi)-\pi H(\xi)\right. \\
\left.-p(\xi)\left((1-\varpi) L_{\mathcal{L}}(\xi)+\varpi L_{\mathcal{H}}(\xi)\right)\right) d \xi \\
H(s)-H(t) \leq \int_{t}^{s}\left((r+p(\xi)) H(\xi)-r b u^{L}(\xi)-p(\xi) H_{\mathcal{H}}(\xi)\right) d \xi \quad \text { for all } t<s .
\end{gathered}
$$

\footnotetext{
${ }^{7}$ The auditing cost is less than $e^{-\pi \epsilon} \sum_{j=1}^{J} \gamma \varpi^{j-1}+\left(1-e^{-\pi \epsilon}\right) J \gamma \leq \gamma /(1-\varpi)+\left(1-e^{-\pi \epsilon}\right) J \gamma$, which remains finite for large $J$ because $\epsilon$ can be made arbitrarily small.
} 
Note that the above integral equations are generalized versions of (4) and (5) in Section 2. If the auditing technology is not available (i.e., $p(\cdot)=0$ ), then (19) and (20) are identical to (4) and (5).

Remark 4 . In the domain of the cost function, the utility pair $L=H<0$ can be delivered by the contract

$$
\begin{gathered}
L(\cdot)=H(\cdot)=L_{\mathcal{H}}(\cdot)=H_{\mathcal{H}}(\cdot)=L=H, \quad H_{\mathcal{L}}(\cdot) \geq L_{\mathcal{L}}(\cdot)=\frac{1}{2} L \\
c^{H}(\cdot)=c(H), \quad c^{L}(\cdot)=c(L)-\left(w_{H}-w_{L}\right), \quad p(\cdot)=\frac{2 r\left(\left|u\left(w_{L}-w_{H}\right)\right|-1\right)}{1-\varpi} .
\end{gathered}
$$

This contract satisfies (19) and (20).

In the following discussion, we use the same heuristic arguments as in Section 2 to obtain a HJB equation. Similar to (13), the differential equation for $x$ is

$$
\frac{d x}{d t}=r(x-b) \hat{u}^{L}+\pi(x-1) x+p\left(\hat{H}_{\mathcal{H}}-(1-\varpi) x \hat{L}_{\mathcal{L}}-\varpi x \hat{L}_{\mathcal{H}}\right)+\hat{\mu},
$$

where

$$
\hat{u}^{L} \equiv \frac{u^{L}}{L}, \quad \hat{L}_{\mathcal{L}} \equiv-\frac{L_{\mathcal{L}}}{L}, \quad \hat{H}_{\mathcal{L}} \equiv-\frac{H_{\mathcal{L}}}{L}, \quad \hat{L}_{\mathcal{H}} \equiv-\frac{L_{\mathcal{H}}}{L}, \quad \hat{H}_{\mathcal{H}} \equiv-\frac{H_{\mathcal{H}}}{L},
$$

and $\hat{\mu} \geq 0$ is, again, a slack variable. Similar to (14), the HJB equation satisfied by $\hat{c}(\cdot)$ is

$$
\begin{aligned}
& (r+\pi) \hat{c}(x) \\
& =\min _{\hat{u}^{L}, p, \hat{L}_{\mathcal{L}}, \hat{L}_{\mathcal{H}}, \hat{H}_{\mathcal{L}}, \hat{H}_{\mathcal{H}}, \hat{\mu}}\left\{-\frac{1}{\rho}\left(r+\pi-\pi x-r \hat{u}^{L}+p\left((1-\varpi) \hat{L}_{\mathcal{L}}+\varpi \hat{L}_{\mathcal{H}}+1\right)\right)\right. \\
& \quad+\hat{c}^{\prime}(x)\left(r(x-b) \hat{u}^{L}+\pi(x-1) x+p\left(\hat{H}_{\mathcal{H}}-(1-\varpi) x \hat{L}_{\mathcal{L}}-\varpi x \hat{L}_{\mathcal{H}}\right)+\hat{\mu}\right) \\
& \left.\quad+r c\left(-\hat{u}^{L}\right)+\pi c(-x)+p\left((1-\varpi) C\left(\hat{L}_{\mathcal{L}}, \hat{H}_{\mathcal{L}}\right)+\varpi C\left(\hat{L}_{\mathcal{H}}, \hat{H}_{\mathcal{H}}\right)+\gamma-\hat{c}(x)\right)\right\} .
\end{aligned}
$$

Collecting all the terms with $p$, the above HJB equation can be written as

$$
\begin{aligned}
\hat{c}(x)=\frac{1}{r+\pi} \min _{\hat{u}^{L}, \hat{\mu}}\left\{-\frac{1}{\rho}(r+\pi-\right. & \left.\pi x-r \hat{u}^{L}\right)+\hat{c}^{\prime}(x)\left(r(x-b) \hat{u}^{L}+\pi(x-1) x+\hat{\mu}\right) \\
+ & \left.r c\left(-\hat{u}^{L}\right)+\pi c(-x)\right\}+\frac{1}{r+\pi} \min _{p \geq 0} p\left(\gamma-\mathcal{M}\left(\hat{c}^{\prime}(x), x\right)\right),
\end{aligned}
$$

where

$$
\begin{array}{r}
\mathcal{M}(d, x) \equiv \max _{\hat{L}_{\mathcal{L}}, \hat{L}_{\mathcal{H}}, \hat{H}_{\mathcal{L}}, \hat{H}_{\mathcal{H}}}\left\{\frac{1}{\rho}\left((1-\varpi) \hat{L}_{\mathcal{L}}+\varpi \hat{L}_{\mathcal{H}}+1\right)+d\left((1-\varpi) x \hat{L}_{\mathcal{L}}+\varpi x \hat{L}_{\mathcal{H}}-\hat{H}_{\mathcal{H}}\right)\right. \\
\left.+\left(\hat{c}(x)-(1-\varpi) C\left(\hat{L}_{\mathcal{L}}, \hat{H}_{\mathcal{L}}\right)-\varpi C\left(\hat{L}_{\mathcal{H}}, \hat{H}_{\mathcal{H}}\right)\right)\right\}
\end{array}
$$


measures the benefit of an audit. Note that the benefit and, hence, the decision whether to audit, do not depend on $H$ and $L$ separately, but depend only on the ratio $x$. Auditing yields two benefits: $\hat{c}(x)-(1-\varpi) C\left(\hat{L}_{\mathcal{L}}, \hat{H}_{\mathcal{L}}\right)-\varpi C\left(\hat{L}_{\mathcal{H}}, \hat{H}_{\mathcal{H}}\right)$ is the benefit due to the jumps to new states that potentially have lower cost, while $d\left((1-\varpi) x \hat{L}_{\mathcal{L}}+\varpi x \hat{L}_{\mathcal{H}}-\right.$ $\left.\hat{H}_{\mathcal{H}}\right)+(1 / \rho)\left((1-\varpi) \hat{L}_{\mathcal{L}}+\varpi \hat{L}_{\mathcal{H}}+1\right)$ measures the benefit from relaxing the incentive constraint and the promise-keeping constraint through auditing (because auditing changes the evolution of both $x(t)$ and $L(t))$.

The HJB equation requires that

$$
\mathcal{M}\left(\hat{c}^{\prime}(x), x\right) \leq \gamma,
$$

with $p=0$ whenever the inequality is strict. Hence $\min _{p \geq 0} p\left(\gamma-\mathcal{M}\left(\hat{c}^{\prime}(x), x\right)\right)=0$ and auditing does not contribute to the right side of the HJB equation. Therefore,

$$
\begin{aligned}
\hat{c}(x)=\frac{1}{r+\pi} \min _{\hat{u}} \hat{u}_{\hat{\mu}}\left\{-\frac{1}{\rho}(r\right. & \left.+\pi-\pi x-r \hat{u}^{L}\right) \\
& \left.+\hat{c}^{\prime}(x)\left(r(x-b) \hat{u}^{L}+\pi(x-1) x+\hat{\mu}\right)+r c\left(-\hat{u}^{L}\right)+\pi c(-x)\right\},
\end{aligned}
$$

which is identical to (14), except here $\hat{c}(\cdot)$ is also defined at the boundary $x=1$.

In Section 3.1, we solve for the optimal contract. In the solution, the principal audits if and only if $x=1$ and the optimal auditing is periodic. In Section 3.2, we show that the principal would not use atomic auditing. In Section 3.3, we study implications of the optimal contract. Finally, in Section 3.4, we discuss various extensions of the model.

\subsection{The optimal contract with auditing}

3.1.1 Candidate solution Recall that the candidate solution in Section 2 starts with the initial condition $\left(x^{*}, \mathcal{H}\left(0, x^{*}\right)\right)$. The principal's cost when the auditing technology is available must be less than that in Section 2. Hence in solving the ordinary differential equation (ODE) (16), we focus on solutions that start with $x_{0}>x^{*}$ and hit the boundary $x=1$.

The auditing arrival rate $p$ is positive when $x=1$. To see this, suppose $p=0$ when $x=1$. Then

$$
\left.\frac{d x}{d t}\right|_{x=1}=r(1-b) \hat{u}^{L}+\hat{\mu}>0,
$$

which violates the domain restriction $x \leq 1$ (i.e., $L \leq H)$. Therefore, $p\left(\gamma-\mathcal{M}\left(\hat{c}^{\prime}(x), x\right)\right)=$ 0 implies

$$
\left.\mathcal{M}\left(\hat{c}^{\prime}(x), x\right)\right|_{x=1}=\gamma .
$$

With this condition, there is a unique solution to (16). We verify the optimality of the candidate solution in Section 3.1.2.

Lemma 6. There is a unique initial condition $x^{* *}$ such that the solution $\hat{c}^{x^{* *}}(\cdot)$ to (16) satisfies $\mathcal{M}\left(\left(\hat{c}^{x^{* *}}\right)^{\prime}(1), 1\right)=\gamma$ (see Figure 2). 


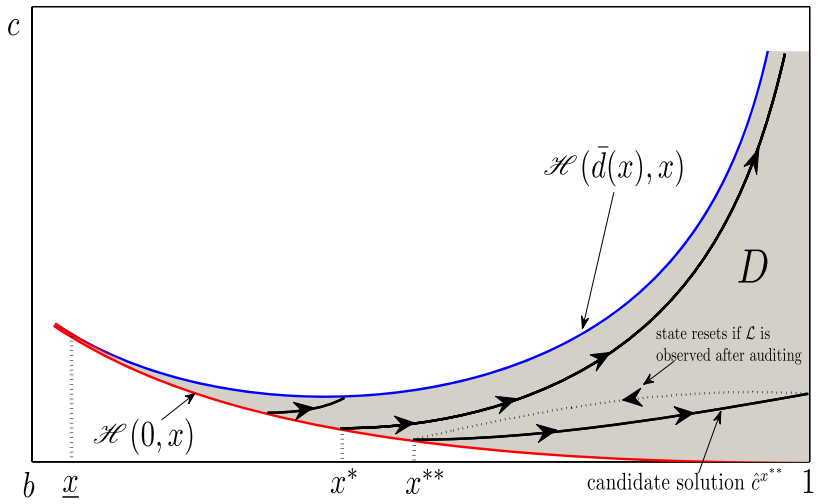

FIgURE 2. The candidate solution with auditing.

Next we show that the benefit of auditing is monotonic in $x$.

Lemma 7. The benefit function $\mathcal{M}\left(\left(\hat{c}^{x^{* *}}\right)^{\prime}(x), x\right)$ increases in $x$. Therefore, if $x<1$, then

$$
\mathcal{M}\left(\left(\hat{c}^{x^{* *}}\right)^{\prime}(x), x\right)<\gamma .
$$

The intuition for the monotonicity of the benefit function can be seen in a simple case. Suppose that auditing is perfect and that the principal pre-commits to auditing at time $t$ with probability 1 . Then the high-income agent cannot underreport at $t$, and the principal can jump from state $(L, H)$ to $\left(L, L x^{* *}\right)$ without violating any incentive constraint. Here the benefit of auditing is $C(L, H)-C\left(L, L x^{* *}\right)=\hat{c}^{x^{* *}}(x)-\hat{c}^{x^{* *}}\left(x^{* *}\right)$, which increases in $x$. In other words, the benefit here is to reset $H$ and remove the distortion in the consumption allocation. The larger is the distortion, the larger is the benefit of auditing. In the general case of stochastic auditing with errors, the benefit of auditing is similar, although $\mathcal{M}$ takes a more complicated form.

Let $N$ be the moment when the trajectory of $x(t)$ implied by the candidate solution reaches the boundary 1 starting from $x(0)=x^{* *}$. In the pre-commitment contract, the principal guarantees not to audit until $N$, despite the fact that income is private information. When the duration of the agent's low-income reports reaches $N$, he is audited randomly according to an endogenous arrival rate $p>0$. The actual instant of audit depends on the realization of the audit random variable, so the actual audit could be realized at any $t \geq N$. Two remarks regarding $N$ are in order: (i) while auditing is stochastic, the threshold duration $N$ is deterministic; (ii) while $N$ depends on primitives of the model, it does not depend on the initial promised utility $L_{0}$. The homogeneity property implies that if $\{(L(t), H(t)) ; t \geq 0\}$ is the optimal trajectory when the initial promise is $L_{0}$, then $\left\{\left(\left(\bar{L}_{0} / L_{0}\right) L(t),\left(\bar{L}_{0} / L_{0}\right) H(t)\right) ; t \geq 0\right\}$ is the optimal trajectory when the initial promise is $\bar{L}_{0} \neq L_{0}$. The two paths reach $x=1$ at the same time.

Our main result is that the auditing pattern is periodic. The optimal mechanism consists of cycles. A low-income agent begins each cycle with continuation utilities $(L, H)$, such that $H / L=x^{* *}$, and is initially not audited. The pair $(L(\cdot), H(\cdot))$ falls with the duration of low-income reports until $N$ (i.e., until $x=1$ ). After $N$, the principal randomly 
audits and $(L(\cdot), H(\cdot))$ remains unchanged until audit arrives. When the audit arrives and the observed signal is $\mathcal{H}$, the agent is punished by a downward jump in his continuation utilities. The new state still satisfies $x=1$ and, hence, the agent continues to be audited randomly. A new cycle starts the moment after the observed signal from the audit is $\mathcal{L}$. In the new cycle, the low-income agent begins with improved continuation utilities $\left(L_{\mathcal{L}}, H_{\mathcal{L}}\right)$, such that $H_{\mathcal{L}} / L_{\mathcal{L}}=x^{* *}$.

Proposition 1. Given the initial utility $L_{0}$, the principal chooses $H_{0}$ such that $H_{0} / L_{0}=$ $x^{* *}$. Starting from $x^{* *}$, the solution to ODE (16) reaches $x=1$ in finite time $N$. After $N$ and conditional on low-income report, the principal audits with an arrival rate $p>0$. The utility pair $(L(t), H(t))$ remains stationary: $L(t)=H(t)=L(N)=H(N) \forall t \geq N$ until the agent is randomly drawn to be audited. If the observed signal from the audit is $\mathcal{H}$, the agent is punished, $L_{\mathcal{H}}=H_{\mathcal{H}}<L(N)$. If the signal is $\mathcal{L}$, the agent is rewarded, $L_{\mathcal{L}}>L(N)$. The new state satisfies $H_{\mathcal{L}} / L_{\mathcal{L}}=x^{* *}$ and, hence, the contract enters a new cycle.

There are two instruments to provide incentives for truthfully reporting the transition to high income. The first instrument is dynamic taxation that distorts the consumption path; the second instrument is auditing. Proposition 1 shows that the principal uses the first instrument only when $L<H$ and uses the second only when $L=H$. Recall that $\hat{c}(x)$ measures the distortion in the continuation contract (see the discussion after Lemma 2), and the closer is $x$ to 1, the higher is the distortion. The principal switches to the auditing instrument only when the distortion is the highest, i.e., when $L=H$.

The principal alternates between dynamic taxation and auditing because the net benefit of the first instrument is decreasing with the distortion, while that of the second is increasing with the distortion. Starting with the full-insurance consumption path, a first-order distortion in consumption generates only a second-order cost to the principal. Thus, when consumption distortion is small, it is nearly costless to use the first instrument and the principal avoids the second instrument of auditing, which has a cost $\gamma$. When $L=H$, the consumption distortion is high, so further distortion using the dynamic taxation instrument is costly. At this stage, the principal abandons the first instrument and switches to the second. Conditional on discovering the truth-teller after an audit, she removes the previously accumulated distortion by resetting the state variables. After the resetting, a new cycle begins and the principal switches back to the first instrument.

Periodic auditing is the outcome of joint optimization of the two instruments. If distortion does not grow as it does here, then periodic auditing may no longer be optimal. In particular, in models with i.i.d. income, such as Green (1987), the distortion is constant as noted in Section 2.1. Wang (2005) shows that the optimal auditing probability in such environments is time invariant. In a private information model with two-state Markov chain shocks, Nakamura (2009) derives a debt contract that follows a cycle. In the "normal" phase of the cycle, the borrower pays a fixed amount to the lender, and in the "restructuring" phase of the cycle, the borrower declares bankruptcy and his payments are reduced. Unlike our model, the income is not reported in Nakamura's model and the contract is not designed to elicit truthful revelation of income. His result is due 
to the evolution of the lender's beliefs regarding the borrower's income, not due to the accumulation of distortions.

Our periodic auditing result holds no matter how high the auditing cost $\gamma$ is. This is because the distortion in consumption converges to infinity (see Section 2) and the benefit of using the auditing instrument eventually surpasses any finite cost. This result contrasts with those in Wang (2005) and Monnet and Quintin (2005), where the benefit of audit remains bounded and the principal does not audit when $\gamma$ is large.

3.1.2 Verification Similar to Section 2.2.2, we verify that the allocation implied by the candidate solution $\hat{c}^{* *}(\cdot)$ is indeed optimal.

Lemma 8. When the auditing technology is available, the contract implied by $\hat{c}^{* * *}(\cdot)$ is optimal.

\subsection{No atomic auditing}

In the previous subsection, we showed that the principal would set $p=0$ when $L<H$ and would set $p>0$ but finite when $L=H$. In this subsection, we show that the principal never sets $p=\infty$, i.e., she never uses atomic auditing. To see this, suppose that the auditing probability has an atom of size $P>0$ with the state $(L, H)$. With probability $P$, the principal audits, and with probability $1-P$, she does not. Thus, the cost minimization problem for the principal is

$$
\begin{gathered}
M(P)=\min _{L_{\mathcal{L}}, H_{\mathcal{L}}, L_{\mathcal{H}}, H_{\mathcal{H}}, \bar{L}, \bar{H}} P\left((1-\varpi) C\left(L_{\mathcal{L}}, H_{\mathcal{L}}\right)+\varpi C\left(L_{\mathcal{H}}, H_{\mathcal{H}}\right)+\gamma\right)+(1-P) C(\bar{L}, \bar{H}) \\
\end{gathered}
$$

where $\left(L_{\mathcal{L}}, H_{\mathcal{L}}\right)$ denotes the state if the observed signal from the audit is $\mathcal{L},\left(L_{\mathcal{H}}, H_{\mathcal{H}}\right)$ denotes the state if the signal is $\mathcal{H}$, and $(\bar{L}, \bar{H})$ denotes the state if the agent is not audited. Note that $M(0)=C(L, H)$. Lemma 9 below states that atomic auditing is not optimal.

Lemma 9. At any $(L, H), L \leq H$, there is no atomic auditing, because $M(P)>M(0)$ for all $P>0$.

\subsection{Implications of the optimal contract}

1. Reducing the cost of auditing (smaller $\gamma$ ) increases the auditing frequency. A smaller $\gamma$ makes the auditing instrument cheaper. As a result, the principal is willing to audit more frequently. For all $\gamma$, the auditing instrument is used only when $x=1$. However, $x^{* *}$ (see Figure 2) increases with smaller $\gamma$. Hence, $N$, the time taken to reach $x=1$ from $x=x^{* *}$, is less for smaller $\gamma$. 
2. Increasing the variance of income (larger $w_{H}-w_{L}$ ) increases the auditing frequency. With a larger $w_{H}-w_{L}$, there is a larger benefit to the agent from underreporting income. In the absence of an auditing technology, the low-income agent's consumption path needs to be distorted more to provide dynamic incentives for truthtelling. The benefit of auditing is increased with a larger $w_{H}-w_{L}$; as a result, the auditing instrument is used more frequently.

3. Agents with an intermediate level of risk aversion are audited more frequently relative to agents with either low or high risk aversion. When the absolute risk aversion $\rho$ is small, the variation in the consumption path implies little cost to the principal; thus, there is no need to use auditing to reduce the distortion. When $\rho$ is large, a small variation in consumption is able to generate large incentive effects. Again, there is no need to audit. More specifically, let $L_{0}=-1$ and consider a contract in which auditing is not used and consumptions decline linearly, i.e., $c^{H}(t)=c^{L}(t)=(\log (\pi /(r+\pi))-r t) / \rho$. This contract delivers the promised utility $L_{0}$ because

$$
\int_{0}^{\infty} e^{-(r+\pi) t}\left(\pi H(t)+r u^{L}(t)\right) d t=-\int_{0}^{\infty} e^{-(r+\pi) t}\left((\pi+r) e^{r t} \frac{\pi}{r+\pi}\right) d t=-1 .
$$

It is I.C. because

$$
\frac{d H(t)}{d t}=r H(t)<r H(t)-r b u^{L}(t) .
$$

When $\rho \rightarrow \infty$, the cost of the contract converges to the full-information cost $c\left(L_{0}\right)$. The above no-auditing contract has very little distortion in consumption, and there is no need to correct it frequently by auditing.

\subsection{Discussion}

Show-me assumption. We assume that the principal always asks the agent to show his income. This assumption prevents low-income agents from reporting high income; hence, we do not have to include the incentive constraint for the low-income agent. In fact, even if we include this incentive constraint, it never binds. To see this, recall that $x(t)$ always stays above $\underline{x}$, where $\underline{x}$ is the unique root to $r(x-b)+\pi(x-1) x=0$ in $(b, 1)$ (see Lemma 3). Therefore, the incentive constraint for the low-income agent

$$
\int_{t}^{\infty} e^{-(r+\pi)(s-t)}\left(r b^{-1} H(t)+\pi H(t)\right) d s=\frac{r b^{-1}+\pi}{r+\pi} H(t)<L(t)
$$

is always satisfied, because

$$
\frac{r b^{-1}+\pi}{r+\pi} x(t)-1>\frac{r b^{-1}+\pi}{r+\pi} \underline{x}-1=\frac{r}{r+\pi}\left(\underline{x} b^{-1}-1\right)>0 .
$$

Auditing error. If auditing is perfect $(\varpi=0)$, then an arbitrarily small arrival rate of audit plus infinite penalty delivers the full-information constant consumption. Our 
assumption $\varpi>0$ implies that when $\mathcal{H}$ is observed, the principal cannot be 100 percent certain that the agent is a liar and, hence, would not want to impose an infinite penalty.

Finite punishment. When punishment is exogenous and finite, the full-information constant consumption cannot be achieved even with perfect auditing.

In Ravikumar and Zhang (2010), after an audit, a liar is caught with probability 1 and his consumption is reduced forever by an exogenous amount. We show that the optimal mechanism is still periodic auditing.

Popov (2007) imposes an exogenous lower bound on the agent's continuation utility in an i.i.d. income model with no auditing errors. He shows that both the distortion and the auditing probability depend on the level of the agent's continuation utility. This contrasts with our model, where the auditing probability is independent of the low-income agent's continuation utility. In his model, after an audit, the liar is pushed to the lower bound on continuation utility. Thus, in the event of being caught after the audit, agents with high continuation utility suffer a more severe punishment relative to agents with low continuation utility. For agents with high continuation utility, a low auditing probability combined with a severe punishment is very effective. For agents with continuation utility at the lower bound, the auditing instrument is not effective because the principal cannot punish a liar any more. ${ }^{8}$ In our model, there is no lower bound on continuation utility and the auditing instrument is always effective.

The benefit of auditing in our model is to remove the distortion embedded in the dynamic contract. This notion of auditing benefit applies more generally. Consider a model where the agent's private information $\theta \in\left\{\theta_{1}, \theta_{2}, \ldots, \theta_{n}\right\}$ evolves as a Markov chain. The private information $\theta$ could represent the agent's income, his taste shocks, or other risks that are relevant to allocating resources to the agent efficiently. The principal's problem is to deliver an initial level of utility to the agent at a minimal cost, subject to incentive constraints. This problem can be formulated recursively by keeping track of the shock $\theta$ and a vector of continuation utilities $v \in \mathbb{R}^{n}$; the vector $v$ contains the continuation utility, $v_{\theta}$, for the agent whose current type is $\theta$ and $(n-1)$ continuation utilities, $v_{-\theta}$, for agents who just transited to other states. Let $C(v, \theta)$ denote the principal's discounted expected cost of delivering the vector $v$. Suppose that auditing is perfect and nonstochastic, and that the punishment is exogenous and finite (the punishment could be a function of the vector $v$ and the liar's true type, observed after auditing). If no cheating is found upon auditing, the principal can re-optimize all continuation utilities $v_{-\theta}$ and obtain a benefit $C\left(v_{\theta}, v_{-\theta}, \theta\right)-\min _{\tilde{v}_{-\theta}} C\left(v_{\theta}, \tilde{v}_{-\theta}, \theta\right)$. In such a general environment, it is optimal for the principal to wait for some positive amount of time after an audit before she audits again. This is because $v_{-\theta}$ is reset to the minimizer immediately after the audit. Continuity implies that $v_{-\theta}$ remains close to the minimizer for some interval of time. Since auditing has a fixed cost, there is a region around the minimizer where the cost of auditing dominates the benefit. Thus, the optimal contract implies that the principal commits to no auditing for a length of time and eliminates the distortion by resetting the state variable after every audit. The duration between two audits may not be a constant and may depend on how the distortion evolves in the optimal contract.

\footnotetext{
${ }^{8}$ At low continuation utility in Popov's environment, both the dynamic taxation and the auditing instruments are weak, so the determination of auditing probability is nontrivial.
} 
Preferences. With CARA utility, the distortion in our optimal contract is independent of the agent's continuation utility. Instead the distortion depends only on the ratio of the high-income agent's utility $H$ to the low-income agent's utility $L$. For general utilities, the distortion depends on both the level and the ratio. The principal's cost function becomes two dimensional, making it difficult to obtain a full characterization of the optimal contract.

In a model with constant relative risk aversion (CRRA) preferences, we find that the auditing probability is small when the agent's continuation utility is either extremely high or extremely low (see Lemma 13 in the Appendix). This is consistent with the finding in Section 3.3 that the auditing probability is small when the absolute risk aversion is extremely large or extremely small (the absolute risk aversion in CRRA preferences decreases with consumption and with the agent's level of continuation utility).

Absorbing state. Our assumption that the high-income state is permanent allows us to focus on the path with only low-income reports. If the transition rate from high income to low income, $\pi^{\prime}$, is positive, the analysis becomes more complex. Not only does the principal's problem after the transition to high income become nontrivial, but the dynamics before the transition to $w_{H}$ are also altered. For $\pi^{\prime}>0$, the candidate solution to (16) is not easy to find analytically, because the solution curve may reach the upper bound $\mathscr{H}(\bar{d}(\cdot), \cdot)$, and local Lipschitz condition fails there. Our numerical result shows that without auditing and when $\pi^{\prime}$ is small, the ratio $x=H / L$ approaches 1 (i.e., the distortion approaches infinity). Thus, our periodic auditing result in Section 3 still holds when $\pi^{\prime}$ is small. When $\pi^{\prime}$ is much larger than $\pi, x$ still increases with the duration of low-income reports, but it approaches a limit strictly below 1 . That is, the distortion in the contract remains bounded. Hence, it is no longer true that auditing is used eventually regardless of the auditing cost $\gamma$. To summarize, our periodic auditing result requires high persistence of the high-income state, but the high-income state need not be absorbing.

Multiple income levels. Our assumption that income is either $w_{H}$ or $w_{L}$ is restrictive. One implication of this assumption is that auditing occurs only at the lowest income level (i.e., $w_{L}$ ). When there are more than two income levels, the principal might audit any income level below the maximum. If we assume that the only binding incentive constraint when the report is $w_{i}$ is for the agent at the next higher income level $w_{i+1}$, then our measure of distortion remains useful. ${ }^{9}$ The distortion in this case is the ratio of the continuation utility for the agent who just transited to $w_{i+1}$ to that for the agent who remains at $w_{i}$. It would be interesting to examine how the distortion evolves and whether the optimal auditing is still periodic with multiple income levels.

\section{Conclusion}

We study insurance and auditing in a repeated hidden income environment with persistent incomes. A principal, with imperfect ability to audit, designs an optimal taxation

\footnotetext{
${ }^{9}$ Kapicka (2010) and Williams (2011) make this assumption in their private-information models. A general condition on primitives for this assumption to be valid is still unknown.
} 
scheme as well as an optimal auditing scheme. When the agent's absolute risk aversion is constant, we show that it is optimal to audit the low-income agent periodically. The optimal mechanism consists of cycles. Within each cycle, an agent reporting low income is guaranteed that he is not audited until the duration of low-income reports exceeds a threshold. After the threshold is reached, the agent is audited randomly. After the random audit, if the agent is discovered to be a truth-teller, then a new cycle begins.

The low-income agent's consumption path is intertemporally distorted. We measure the distortion as the principal's cost minus the cost of the full-information constant consumption that delivers the same utility to the low-income agent. Unlike the repeated hidden income model with i.i.d. incomes, the distortion in our model increases with the duration of low-income reports. Auditing helps the principal detect who is a truth-teller and who is a cheater. She can thus correct the distortion in the consumption path after an audit. The benefit of auditing increases with the duration of low-income reports, whereas the cost of auditing is constant. Consequently, the principal uses the auditing instrument no matter how high the auditing cost is.

Since auditing is imperfect in our model, the principal cannot detect a liar with certainty and, hence, does not want to impose an infinite penalty. If auditing were perfect, then the optimal mechanism is a small probability of audit plus infinite penalty. This mechanism delivers the full-insurance consumption. ${ }^{10}$ However, with perfect auditing but exogenous and finite penalty, the optimal mechanism is periodic auditing.

\section{APPENDix}

Proof of Lemma 1. We show only the equivalence between (2) and (5), since the equivalence between (1) and (4) can be obtained similarly by replacing the inequalities below with equalities.

Necessity. If (2) holds for all $t<s$, then

$$
\begin{aligned}
& H(t)+\int_{t}^{s}\left(r H(\xi)-r b u^{L}(\xi)\right) d \xi \\
& \geq \int_{t}^{s} e^{-r(\xi-t)} r b u^{L}(\xi) d \xi+e^{-r(s-t)} H(s) \\
& \quad+\int_{t}^{s}\left(r\left(\int_{\xi}^{s} e^{-r(\eta-\xi)} r b u^{L}(\eta) d \eta+e^{-r(s-\xi)} H(s)\right)-r b u^{L}(\xi)\right) d \xi \\
& =\left(e^{-r(s-t)}+\int_{t}^{s} r e^{-r(s-\xi)} d \xi\right) H(s)+\int_{t}^{s}\left(e^{-r(\xi-t)}-1\right) r b u^{L}(\xi) d \xi \\
& \quad+\int_{t}^{s} r\left(\int_{\xi}^{s} e^{-r(\eta-\xi)} r b u^{L}(\eta) d \eta\right) d \xi
\end{aligned}
$$

\footnotetext{
${ }^{10}$ When both the auditing probability and the penalty are exogenously specified, Armenter and Mertens (2010) show that full-insurance consumption can be achieved in the long run in a dynamic Mirrlees taxation model.
} 


$$
\begin{aligned}
& =H(s)+\int_{t}^{s}\left(e^{-r(\xi-t)}-1\right) r b u^{L}(\xi) d \xi+\int_{t}^{s}\left(\int_{t}^{\eta} r e^{-r(\eta-\xi)} d \xi\right) r b u^{L}(\eta) d \eta \\
& =H(s)+\int_{t}^{s}\left(e^{-r(\xi-t)}-1\right) r b u^{L}(\xi) d \xi+\int_{t}^{s}\left(1-e^{-r(\eta-t)}\right) r b u^{L}(\eta) d \eta \\
& =H(s) .
\end{aligned}
$$

Hence, inequality (5) is verified.

Sufficiency. Define an absolutely continuous function $f(\cdot)$ as

$$
f(s) \equiv \int_{t}^{s} e^{-r(\xi-t)} r b u^{L}(\xi) d \xi+e^{-r(s-t)}\left(H(t)+\int_{t}^{s}\left(r H(\xi)-r b u^{L}(\xi)\right) d \xi\right) .
$$

Because $f$ is absolutely continuous, it is differentiable almost everywhere (a.e.), and

$$
\begin{aligned}
f^{\prime}(s)= & e^{-r(s-t)} r b u^{L}(s)-r e^{-r(s-t)}\left(H(t)+\int_{t}^{s}\left(r H(\xi)-r b u^{L}(\xi)\right) d \xi\right) \\
& \quad+e^{-r(s-t)}\left(r H(s)-r b u^{L}(s)\right) \\
= & r e^{-r(s-t)}\left(H(s)-H(t)-\int_{t}^{s}\left(r H(\xi)-r b u^{L}(\xi)\right) d \xi\right) \quad \text { a.e. }
\end{aligned}
$$

If (5) holds, then $f^{\prime}(s) \leq 0$ a.e. Then it follows from Theorem 29.15 in Aliprantis and Burkinshaw (1990) that

$$
f(s)=f(t)+\int_{t}^{s} f^{\prime}(\xi) d \xi \leq f(t)=H(t) .
$$

Therefore,

$$
\int_{t}^{s} e^{-r(\xi-t)} r b u^{L}(\xi) d \xi+e^{-r(s-t)} H(s) \leq f(s) \leq H(t),
$$

which verifies inequality (2).

Proof of Lemma 2. (i) Equation (6) holds because $\left\{\left(c^{L *}(t), c^{H *}(t)\right) ; t \geq 0\right\}$ is the optimal contract to implement $(L, H)$ if and only if $\left\{\left(c^{L *}(t)-\log (\alpha) / \rho\right.\right.$, $\left.\left.c^{H *}(t)-\log (\alpha) / \rho\right) ; t \geq 0\right\}$ is the optimal contract to implement $(\alpha L, \alpha H)$.

(ii) We show that $C(L, \bar{H}) \leq C(L, H)$ for any $H<\bar{H}$. Pick any I.C. contract $\left\{\left(c^{L}(t)\right.\right.$, $\left.\left.c^{H}(t)\right) ; t \geq 0\right\}$ that implements $(L, H)$. If we simply redefine $c^{H}(0)$ to be $c(\bar{H})$ and leave other consumptions unchanged, the contract implements $(L, \bar{H})$. That is, the modified contract starting with $(L, \bar{H})$ coincides with the original contract immediately after time zero. Hence, the two contracts have the same cost. The modified contract is incentive compatible too, because incentive constraints allow for downward jumps in $H(\cdot)$. Therefore, $C(L, \bar{H}) \leq C(L, H)$.

Proof of Lemma 3. (i) When $\hat{c}^{\prime}(x)=0$, the optimal $\hat{u}^{L}$ is 1 and, hence, $\mathscr{H}(0, x)=$ $(\pi /(r+\pi))(-(1 / \rho)(1-x)+c(-x))$ decreases in $x$. 
(ii) The envelope theorem implies that $(r+\pi)(\partial \mathcal{H}(d, x) / \partial d)=r(x-b) \hat{u}^{L}+$ $\pi(x-1) x=d x / d t$. When $x \leq b$, it follows from $\hat{u}^{L}>0$ and $r(x-b) \hat{u}^{L}+$ $\pi(x-1) x \leq 0$ that $\bar{d}(x)=0$. When $x>b$, the first-order condition implies that the optimal policy $\hat{u}^{L}(d)$ is $1 /(1+d \rho(x-b))$ and decreases in $d$. Hence

$$
(r+\pi) \frac{\partial \mathscr{H}(d, x)}{\partial d}=\frac{r(x-b)}{1+d \rho(x-b)}+\pi(x-1) x,
$$

which decreases in $d$ because $x>b$. Thus $\mathscr{H}(\cdot, x)$ is concave in $d$ and to find $\bar{d}(x)$, it suffices to study the first-order condition. When $d=0$,

$$
\left.(r+\pi) \frac{\partial \mathscr{H}(d, x)}{\partial d}\right|_{d=0}=r(x-b)+\pi(x-1) x\left\{\begin{array}{cc}
\leq 0 & \text { if } b<x \leq \underline{x} \\
>0 & \text { if } \underline{x}<x<1 .
\end{array}\right.
$$

Recall that $\underline{x}$ is the unique root of $r(x-b)+\pi(x-1) x=0$ in $(b, 1)$. From these first-order conditions, we know that the optimal $\bar{d}(x)$ is 0 when $b \leq$ $x \leq \underline{x}$. When $\underline{x}<x<1$, the first-order condition $(r+\pi)(\partial \mathscr{H}(d, x) / \partial d)=$ $r(x-b) /(1+d \rho(x-b))+\pi(x-1) x=0$ yields

$$
\bar{d}(x)=\frac{1}{\rho}\left(\frac{r}{\pi x(1-x)}-\frac{1}{x-b}\right)>0 .
$$

(iii) Part (ii) states that if $x>\underline{x}$, then $\partial \mathscr{H}(d, x) / \partial d=0$ when $d=\bar{d}(x)$ and $\partial \mathscr{H}(d, x) /$ $\partial d>0$ when $0 \leq d<\bar{d}(x)$.

Proof of Lemma 4. (i) Because $d^{*}(x, \hat{c})$ in (16) is continuously differentiable in the open set $D$, it satisfies the local Lipschitz condition at every point $(x, \hat{c})$. Hence, the solution to (16) exists and is unique in a neighborhood of every point. ${ }^{11}$ Then a global solution can be obtained by pasting the local solutions until the solution reaches boundaries of $D$.

To simplify notation, we omit the superscript $x_{0}$ from $\hat{c}^{x_{0}}(\cdot)$ in the following discussion. To show that $\hat{c}(\cdot)$ is strictly convex, it suffices to show that $\hat{c}^{\prime \prime}>\rho\left(\hat{c}^{\prime}\right)^{2} \geq 0$. Differentiating $\hat{c}=\mathscr{H}\left(\hat{c}^{\prime}, x\right)$ with respect to $x$ yields

$$
\begin{aligned}
(r+\pi) \hat{c}^{\prime} & =(r+\pi)\left(\mathscr{H}_{x}+\mathscr{H}_{d} \hat{c}^{\prime \prime}\right) \\
& =\left(\frac{\pi}{\rho}+\hat{c}^{\prime}\left(r \hat{u}^{L}+\pi(2 x-1)\right)-\frac{\pi}{\rho x}\right)+\left(r(x-b) \hat{u}^{L}+\pi(x-1) x\right) \hat{c}^{\prime \prime} \\
& <\hat{c}^{\prime}\left(r \hat{u}^{L}+\pi\right)+\left(r(x-b) \hat{u}^{L}+\pi(x-1) x\right) \hat{c}^{\prime \prime},
\end{aligned}
$$

where the inequality follows from $x<1$ and $\hat{c}^{\prime}>0$. Then it follows from $\hat{u}^{L}=$ $1 /\left(1+\rho(x-b) \hat{c}^{\prime}\right)$ that

$$
\hat{c}^{\prime \prime}(x)>\frac{r \hat{c}^{\prime}-r \hat{c}^{\prime} \hat{u}^{L}}{r(x-b) \hat{u}^{L}+\pi(x-1) x}>\frac{r \hat{c}^{\prime}-r \hat{c}^{\prime} \hat{u}^{L}}{r(x-b) \hat{u}^{L}}=\rho\left(\hat{c}^{\prime}\right)^{2} .
$$

\footnotetext{
${ }^{11}$ Although the local Lipschitz condition fails on the upper boundary $\mathscr{H}(\bar{d}(\cdot), \cdot)$ of $D$, our proof does not require it.
} 
To see the strict convexity of $C(L, H)=c(L)+\hat{c}(H / L)$, note that

$$
\begin{aligned}
C_{L L} & =\rho^{-1} L^{-2}+2 \hat{c}^{\prime} H L^{-3}+\hat{c}^{\prime \prime} H^{2} L^{-4}>0 \\
C_{H H} & =\hat{c}^{\prime \prime} L^{-2}>0 \\
C_{H L} & =-\hat{c}^{\prime} L^{-2}-\hat{c}^{\prime \prime} H L^{-3} .
\end{aligned}
$$

Therefore,

$$
C_{L L} C_{H H}-C_{H L}^{2}=\rho^{-1} L^{-2} \hat{c}^{\prime \prime} L^{-2}-\left(-\hat{c}^{\prime} L^{-2}\right)^{2}>0 .
$$

To see that distortion increases with the duration of low-income reports, recall from the proof of part (ii) in Lemma 3 that $d x / d t=(r+\pi)(\partial \mathscr{H}(d, x) / \partial d)>0$ when $(x, \hat{c}(x)) \in D$.

(ii) First, we show that solutions are ordered. If $x_{0}$ and $y_{0}$ are two initial conditions such that $x_{0}<y_{0}$, then $\hat{c}^{x_{0}}(x)>\hat{c}^{y_{0}}(x)$ whenever both $\hat{c}^{x_{0}}(\cdot)$ and $\hat{c}^{y_{0}}(\cdot)$ are defined at $x$. Initially when $x=y_{0}$, since $\hat{c}^{x_{0}}(\cdot)$ is increasing, $\hat{c}^{x_{0}}\left(y_{0}\right)>\hat{c}^{x_{0}}\left(x_{0}\right)>\hat{c}^{y_{0}}\left(y_{0}\right)$. When $x>y_{0}$, because $d^{*}(x, c)$ increases in $c$, we get $d^{*}\left(x, \hat{c}^{x_{0}}(x)\right)>d^{*}\left(x, \hat{c}^{y_{0}}(x)\right)$. That is, curve $\hat{c}^{x_{0}}(\cdot)$ rises faster than $\hat{c}^{y_{0}}(\cdot)$. Consequently $\hat{c}^{x_{0}}(\cdot)$ is always above $\hat{c}^{y_{0}}(\cdot)$.

Second, we show the existence of an initial condition $x^{*}$ such that the solution $\hat{c}^{x^{*}}(\cdot)$ does not exit $D$. Let $A$ be the collection of initial conditions such that the solution exits $D$ for the first time at $x=1$,

$$
A \equiv\left\{x_{0} \in(\underline{x}, 1): \hat{c}^{x_{0}}(x)<\mathscr{H}(\bar{d}(x), x) \forall x \in\left[x_{0}, 1\right) \text { and } \hat{c}^{x_{0}}(1) \in \mathbb{R}\right\} .
$$

Clearly, (i) $A$ is nonempty since $x_{0} \in A$ when $x_{0}$ is sufficiently close to 1 , and (ii) $A$ does not contain all points in $(\underline{x}, 1)$ because the solution $\hat{c}^{x_{0}}(\cdot)$ will reach the upper boundary when $x_{0}$ is sufficiently close to $\underline{x}$. Define $x^{*} \equiv \inf A>\underline{x}$. We now show that the solution $\hat{c}^{x^{*}}(\cdot)$ does not exit $D$. Clearly, $\hat{c}^{x^{*}}(\cdot)$ could not reach $x=1$ because it is above all solutions that reach $x=1$. To prove that $\hat{c}^{x^{*}}(\cdot)$ could not reach the upper boundary $\mathcal{H}(\bar{d}(\cdot), \cdot)$, suppose to the contrary that $\hat{c}^{x^{*}}(x)=\mathscr{H}(\bar{d}(x), x)$ for some $x \in(\underline{x}, 1)$. Then the upper boundary is flatter than $\hat{c}^{x^{*}}(\cdot)$ at $x$, because

$$
\begin{aligned}
\frac{d \mathcal{H}(\bar{d}(x), x)}{d x} & =\frac{\partial \mathcal{H}(\bar{d}(x), x)}{\partial x} \\
& =\frac{1}{r+\pi}\left(\frac{\pi}{\rho}+\bar{d}(x)\left(r \hat{u}^{L}+\pi(2 x-1)\right)-\frac{\pi}{\rho x}\right) \\
& <\frac{1}{r+\pi} \bar{d}(x)\left(r \hat{u}^{L}+\pi(2 x-1)\right) \\
& =\bar{d}(x)=\left(\hat{c}^{x^{*}}\right)^{\prime}(x),
\end{aligned}
$$

since $x<1$ and $\hat{u}^{L}<1$. By continuity, the solution $\hat{c}^{x^{*}+\epsilon}(\cdot)$ must cross the upper boundary when $\epsilon>0$ is sufficiently small. This contradicts the fact that $x^{*}$ is the infimum of $A$ (i.e., $\hat{c}^{x^{*}+\epsilon}(\cdot)$ must be below the upper boundary for all $\epsilon>0$ ). 
Third, we show the uniqueness of $x^{*}$. To the contrary, suppose that both $\hat{c}^{x_{0}}(\cdot)$ and $\hat{c}^{y_{0}}(\cdot)$ stay in $D$ forever for some $x_{0}<y_{0}$. Denote $x_{1}(c)$ and $y_{1}(c)$ as the inverse of $\hat{c}^{x_{0}}(\cdot)$ and $\hat{c}^{y_{0}}(\cdot)$, respectively. That is, $\hat{c}^{x_{0}}\left(x_{1}(c)\right)=\hat{c}^{y_{0}}\left(y_{1}(c)\right)=c$. Since $\lim _{x \rightarrow 1} \mathscr{H}(\bar{d}(x), x)=\lim _{x \rightarrow 1} \hat{c}^{x_{0}}(x)=\lim _{x \rightarrow 1} \hat{c}^{y_{0}}(x)=\infty$, we get that

$$
\lim _{c \rightarrow \infty}\left(y_{1}(c)-x_{1}(c)\right)=0 .
$$

If $x$ is close to 1 and $\bar{d}(x)$ is large, then $(r+\pi) \mathscr{H}_{x}=\pi / \rho+\bar{d}(x)\left(r \hat{u}^{L}+\pi(2 x-1)\right)-$ $\pi /(\rho x)>0$, hence $d^{*}(x, c)$ decreases in $x$, as $\partial d^{*}(x, c) / \partial x=-\mathscr{H}_{x} / \mathscr{H}_{d}<0$. Therefore,

$$
y_{1}^{\prime}(c)-x_{1}^{\prime}(c)=\frac{1}{\left(\hat{c}^{y_{0}}\right)^{\prime}\left(y_{1}\right)}-\frac{1}{\left(\hat{c}^{x_{0}}\right)^{\prime}\left(x_{1}\right)}=\frac{1}{d^{*}\left(y_{1}, c\right)}-\frac{1}{d^{*}\left(x_{1}, c\right)}>0,
$$

which contradicts (24).

Proof of Lemma 5. To see that the cost of the constructed contract is $C(L, H)$, define

$$
f(T) \equiv \int_{0}^{T} e^{-(r+\pi) t}\left(\pi c(H(t))+r c^{L}(t)\right) d t+e^{-(r+\pi) T} C(L(T), H(T)),
$$

which is differentiable because the trajectories $L(\cdot)$ and $H(\cdot)$ implied by $\hat{c}(\cdot)$ are differentiable in $T .{ }^{12}$ It follows from the HJB equation (12) that

$$
\begin{aligned}
f^{\prime}(T)= & e^{-(r+\pi) T}\left(\pi c(H(T))+r c^{L}(T)-C_{L} \frac{d L(T)}{d T}-C_{H} \frac{d H(T)}{d T}\right) \\
& \quad-e^{-(r+\pi) T}(r+\pi) C(L(T), H(T)) \\
= & 0 .
\end{aligned}
$$

Hence $f(\cdot)$ is a constant. In particular, for all $T>0$,

$$
C(L, H)=f(0)=\int_{0}^{T} e^{-(r+\pi) t}\left(\pi c(H(t))+r c^{L}(t)\right) d t+e^{-(r+\pi) T} C(L(T), H(T)) .
$$

Lemma 11 (later in the Appendix) shows that the tail in the preceding equality converges to zero when $T \rightarrow \infty$; thus, taking limit $T \rightarrow \infty$ yields

$$
C(L, H)=\int_{0}^{\infty} e^{-(r+\pi) t}\left(\pi c(H(t))+r c^{L}(t)\right) d t .
$$

To see that the cost of an I.C. contract $\left\{\left(\tilde{c}^{H}(t), \tilde{c}^{L}(t)\right) ; t \geq 0\right\}$ is higher than $C(L, H)$, define

$$
f(T) \equiv \int_{0}^{T} e^{-(r+\pi) t}\left(\pi c(\tilde{H}(t))+r \tilde{c}^{L}(t)\right) d t+e^{-(r+\pi) T} X(T),
$$

\footnotetext{
${ }^{12}$ The trajectory $H(\cdot)$ is differentiable except for a possible downward jump at $t=0$ if $H(0)$ is too large. The possible jump does not affect the following claim that $f(\cdot)$ is a constant, as it does not change the value of the cost function.
} 
where

$$
\begin{array}{r}
X(T) \equiv C(L(0), H(0))+\int_{0}^{T} C_{L}(\tilde{L}(t), \tilde{H}(t))\left((r+\pi) \tilde{L}(t)-\pi \tilde{H}(t)-r \tilde{u}^{L}(t)\right) d t \\
+\int_{0}^{T} C_{H}(\tilde{L}(t), \tilde{H}(t))\left(r \tilde{H}(t)-r b \tilde{u}^{L}(t)\right) d t .
\end{array}
$$

We claim that

$$
X(t) \leq C(\tilde{L}(t), \tilde{H}(t)) \quad \text { for all } t \geq 0 .
$$

If $\tilde{H}(\cdot)$ is differentiable, this is easy to show as $X(0)=C(L(0), H(0))$ and

$$
\begin{aligned}
X^{\prime}(t)= & C_{L}(\tilde{L}(t), \tilde{H}(t))\left((r+\pi) \tilde{L}(t)-\pi \tilde{H}(t)-r \tilde{u}^{L}(t)\right) \\
& \quad+C_{H}(\tilde{L}(t), \tilde{H}(t))\left(r \tilde{H}(t)-r b \tilde{u}^{L}(t)\right) \\
\leq & C_{L}(\tilde{L}(t), \tilde{H}(t))\left((r+\pi) \tilde{L}(t)-\pi \tilde{H}(t)-r \tilde{u}^{L}(t)\right)+C_{H}(\tilde{L}(t), \tilde{H}(t)) \frac{d \tilde{H}(t)}{d t} \\
= & \frac{d C(\tilde{L}(t), \tilde{H}(t))}{d t} .
\end{aligned}
$$

Since we allow for discrete jumps in $\tilde{H}(\cdot)$, we prove (25) in Lemma 12 (later in the Appendix) without assuming the differentiability of $\tilde{H}$. It follows from the HJB equation (12) that

$$
\begin{aligned}
f^{\prime}(T) \geq & e^{-(r+\pi) T}\left(\pi c(\tilde{H}(T))+r \tilde{c}^{L}(T)-C_{L} \frac{d \tilde{L}(T)}{d T}-C_{H}\left(r \tilde{H}(T)-r b \tilde{u}^{L}(T)\right)\right) \\
& \quad-e^{-(r+\pi) T}(r+\pi) X(T) \\
\geq & e^{-(r+\pi) T}\left(\pi c(\tilde{H}(T))+r \tilde{c}^{L}(T)-C_{L} \frac{d \tilde{L}(T)}{d T}-C_{H}\left(r \tilde{H}(T)-r b \tilde{u}^{L}(T)\right)\right) \\
& \quad-e^{-(r+\pi) T}(r+\pi) C(\tilde{L}(t), \tilde{H}(t)) \\
\geq 0 & \quad \text { a.e. }
\end{aligned}
$$

The absolute continuity of $f$ and the nonnegativity of $f^{\prime}(\cdot)$ imply that $f$ is increasing in $T$. Therefore, for all $T$,

$$
\begin{aligned}
C(L, H) & =f(0) \leq f(T) \\
& \leq \int_{0}^{T} e^{-(r+\pi) t}\left(\pi c(\tilde{H}(t))+r \tilde{c}^{L}(t)\right) d t+e^{-(r+\pi) T} C(\tilde{L}(T), \tilde{H}(T)) .
\end{aligned}
$$

Taking limit $T \rightarrow \infty$ and applying Lemma 11 yield

$$
C(L, H) \leq \int_{0}^{\infty} e^{-(r+\pi) t}\left(\pi c(\tilde{H}(t))+r \tilde{c}^{L}(t)\right) d t,
$$

completing the proof. 
Proof of Lemma 6 . We show that $\mathcal{M}\left(\left(\hat{c}^{x_{0}}\right)^{\prime}(1), 1\right)$ is monotonically decreasing in the initial condition $x_{0}$ and $\mathcal{M}\left(\hat{c}^{\prime}(x), x\right)$ can be rewritten as

$$
\begin{aligned}
(1-\varpi) \max _{\hat{L}_{\mathcal{L}}, \hat{H}_{\mathcal{L}}}\left\{\frac{1}{\rho}\left(\hat{L}_{\mathcal{L}}+1\right)+\left(\hat{c}^{x_{0}}\right)^{\prime}(x) x\left(\hat{L}_{\mathcal{L}}+1\right)-C\left(\hat{L}_{\mathcal{L}}, \hat{H}_{\mathcal{L}}\right)+\hat{c}^{x_{0}}(x)\right\} \\
+\varpi \max _{\hat{L}_{\mathcal{H}}, \hat{H}_{\mathcal{H}}}\left\{\frac{1}{\rho}\left(\hat{L}_{\mathcal{H}}+1\right)+\left(\hat{c}^{x_{0}}\right)^{\prime}(x) x\left(\hat{L}_{\mathcal{H}}+1\right)\right. \\
\left.-\frac{\left(\hat{c}^{x_{0}}\right)^{\prime}(x)}{\varpi}\left(\hat{H}_{\mathcal{H}}+x\right)-C\left(\hat{L}_{\mathcal{H}}, \hat{H}_{\mathcal{H}}\right)+\hat{c}^{x_{0}}(x)\right\} .
\end{aligned}
$$

When $x=1$, the first maximization problem is

$$
\begin{aligned}
\max _{\hat{L}_{\mathcal{L}}, \hat{H}_{\mathcal{L}}}\left\{\frac{1}{\rho}\left(\hat{L}_{\mathcal{L}}+1\right)\right. & \left.+\left(\hat{c}^{x_{0}}\right)^{\prime}(1)\left(\hat{L}_{\mathcal{L}}+1\right)-C\left(\hat{L}_{\mathcal{L}}, \hat{H}_{\mathcal{L}}\right)+\hat{c}^{x_{0}}(1)\right\} \\
& =\max _{\hat{L}_{\mathcal{L}}}\left\{\frac{1}{\rho}\left(\hat{L}_{\mathcal{L}}+1\right)+\left(\hat{c}^{x_{0}}\right)^{\prime}(1)\left(\hat{L}_{\mathcal{L}}+1\right)-c\left(\hat{L}_{\mathcal{L}}\right)\right\}-\hat{c}^{x_{0}}\left(x_{0}\right)+\hat{c}^{x_{0}}(1) .
\end{aligned}
$$

The optimal $\hat{L}_{\mathcal{L}}$ is

$$
\hat{L}_{\mathcal{L}}=-\frac{1}{1+\rho\left(\hat{c}^{x_{0}}\right)^{\prime}(1)}>-1 .
$$

Both $\hat{c}^{x_{0}}(1)$ and $\left(\hat{c}^{x_{0}}\right)^{\prime}(1)$ decrease in the initial condition $x_{0}$. It follows from $\left(\hat{L}_{\mathcal{L}}+1\right)>0$ and the envelope theorem that $\max _{\hat{L}_{\mathcal{L}}}\left\{(1 / \rho)\left(\hat{L}_{\mathcal{L}}+1\right)+\left(\hat{c}^{x_{0}}\right)^{\prime}(1)\left(\hat{L}_{\mathcal{L}}+1\right)-c\left(\hat{L}_{\mathcal{L}}\right)\right\}$ increases in $\left(\hat{c}^{x_{0}}\right)^{\prime}(1)$ and hence decreases in $x_{0}$. Further, $\hat{c}^{x_{0}}(1)-\hat{c}^{x_{0}}\left(x_{0}\right)$ decreases with $x_{0}$, because all solutions are ordered by $x_{0}$. Therefore, the maximized value in the first problem decreases with $x_{0}$.

When $x=1$, the second maximization problem is

$$
\max _{\hat{L}_{\mathcal{H}}, \hat{H}_{\mathcal{H}}}\left\{\frac{1}{\rho}\left(\hat{L}_{\mathcal{H}}+1\right)+\left(\hat{c}^{x_{0}}\right)^{\prime}(1)\left(\hat{L}_{\mathcal{H}}+1\right)-\frac{\left(\hat{c}^{x_{0}}\right)^{\prime}(1)}{\varpi}\left(\hat{H}_{\mathcal{H}}+1\right)-C\left(\hat{L}_{\mathcal{H}}, \hat{H}_{\mathcal{H}}\right)+\hat{c}^{x_{0}}(1)\right\} .
$$

We first show that the optimal solution is on the boundary (i.e., satisfies $\hat{L}_{\mathcal{H}}=\hat{H}_{\mathcal{H}}$ ). Suppose not. Then the first-order conditions for an interior solution are

$$
\begin{gathered}
C_{L}\left(\hat{L}_{\mathcal{H}}, \hat{H}_{\mathcal{H}}\right)=\frac{1}{\rho}+\left(\hat{c}^{x_{0}}\right)^{\prime}(1)=C_{L}(-1,-1) \\
C_{H}\left(\hat{L}_{\mathcal{H}}, \hat{H}_{\mathcal{H}}\right)=-\frac{\left(\hat{c}^{x_{0}}\right)^{\prime}(1)}{\varpi}<C_{H}(-1,-1) .
\end{gathered}
$$

Equation (29) and $d C_{L}(t) / d t=0$ (proven in Lemma 10) imply that $\left(\hat{L}_{\mathcal{H}}, \hat{H}_{\mathcal{H}}\right)$ is on a path that eventually reaches $(-1,-1)$. This contradicts (30) and $d C_{H}(t) / d t<0$ (proven in Lemma 10). Therefore, impose $\hat{L}_{\mathcal{H}}=\hat{H}_{\mathcal{H}}$ and rewrite the second maximization problem as

$$
\max _{\hat{L}_{\mathcal{H}}}\left\{\frac{1}{\rho}\left(\hat{L}_{\mathcal{H}}+1\right)+\left(\hat{c}^{x_{0}}\right)^{\prime}(1)\left(\hat{L}_{\mathcal{H}}+1\right)-\frac{\left(\hat{c}^{x_{0}}\right)^{\prime}(1)}{\varpi}\left(\hat{L}_{\mathcal{H}}+1\right)-c\left(\hat{L}_{\mathcal{H}}\right)\right\}
$$


The optimal $\hat{L}_{\mathcal{H}}$ satisfies

$$
\hat{L}_{\mathcal{H}}=-\frac{1}{1+\rho(1-1 / \varpi)\left(\hat{c}^{x_{0}}\right)^{\prime}(1)}<-1
$$

Hence the maximized value in the second problem increases in $\left(\hat{c}^{x_{0}}\right)^{\prime}(1)$ and decreases in $x_{0}$.

We conclude that $\mathcal{M}\left(\left(\hat{c}^{x_{0}}\right)^{\prime}(1), 1\right)$ is monotonically decreasing in $x_{0}$. Hence the candidate solution is unique. The existence proof follows easily from the intermediate value theorem and is omitted.

Proof of Lemma 7. To simplify notation, we omit the superscript $x^{* *}$ from $\hat{c}^{x^{* *}}(\cdot)$ in this proof. Choose a trajectory $(L(t), H(t))$ and let $x(t)=H(t) / L(t)$. Since

$$
\begin{aligned}
C_{L}(L, H) & =-\frac{1}{\rho L}-\frac{\hat{c}^{\prime}(x) x}{L} \\
C_{H}(L, H) & =-\frac{\hat{c}^{\prime}(x)}{L},
\end{aligned}
$$

we can rewrite $\mathcal{M}\left(\hat{c}^{\prime}(x(t)), x(t)\right)$ in (27) as

$$
\begin{aligned}
& (1-\varpi) \max _{L_{\mathcal{L}}, H_{\mathcal{L}}}\left\{C(L(t), H(t))-C_{L}\left(L(t)-L_{\mathcal{L}}\right)-C\left(L_{\mathcal{L}}, H_{\mathcal{L}}\right)\right\} \\
& +\varpi \max _{L_{\mathcal{H}}, H_{\mathcal{H}}}\left\{C(L(t), H(t))-C_{L}\left(L(t)-L_{\mathcal{H}}\right)-\frac{C_{H}}{\varpi}\left(H(t)-H_{\mathcal{H}}\right)-C\left(L_{\mathcal{H}}, H_{\mathcal{H}}\right)\right\} .
\end{aligned}
$$

Because $d x / d t>0$, it is equivalent to show that $\mathcal{M}\left(\hat{c}^{\prime}(x(t)), x(t)\right)$ increases in $t$. Lemma 10 states that $d C_{L}(t) / d t=0$ and $d C_{H}(t) / d t<0$. Applying the envelope theorem, we have

$$
\begin{array}{r}
\frac{d \mathcal{M}}{d t}=(1-\varpi)\left\{C_{L} \frac{d L(t)}{d t}+C_{H} \frac{d H(t)}{d t}-C_{L} \frac{d L(t)}{d t}\right\} \\
+\varpi\left\{C_{L} \frac{d L(t)}{d t}+C_{H} \frac{d H(t)}{d t}-C_{L} \frac{d L(t)}{d t}\right. \\
\left.\quad-\frac{C_{H}}{\varpi} \frac{d H(t)}{d t}-\frac{1}{\varpi} \frac{d C_{H}(t)}{d t}\left(H(t)-H_{\mathcal{H}}\right)\right\} \\
=-\frac{d C_{H}(t)}{d t}\left(H(t)-H_{\mathcal{H}}\right)>0 .
\end{array}
$$

Proof of Proposition 1. Conditional on a signal $\mathcal{L}$, the low-income agent is rewarded, as (28) states that $\hat{L}_{\mathcal{L}}>-1$. Conditional on a signal $\mathcal{H}$, both agents are punished, as (31) states that $\hat{L}_{\mathcal{H}}=\hat{H}_{\mathcal{H}}<-1$.

The trajectory $(L(t), H(t))$ satisfies $L(t)=H(t)$ when $t \geq N$ because $d x / d t>0$ whenever $x<1$. The auditing arrival rate $p$ can be solved from $d L / d t=d H / d t$ at $L=H$, which is

$$
(r+\pi) L-\pi H-r u^{L}-p\left((1-\varpi) L_{\mathcal{L}}+\varpi L_{\mathcal{H}}-L\right)=r H-r b u^{L}-p\left(H_{\mathcal{H}}-H\right) .
$$


Note that when $L=H$,

$$
\begin{aligned}
\frac{H_{\mathcal{H}}-H}{(1-\varpi) L_{\mathcal{L}}+\varpi L_{\mathcal{H}}-L} & =\frac{\hat{H}_{\mathcal{H}}-(-1)}{(1-\varpi) \hat{L}_{\mathcal{L}}+\varpi \hat{L}_{\mathcal{H}}-(-1)} \\
& =\frac{1-\frac{1}{1+(1-1 / \varpi) \rho \hat{c}^{\prime}(1)}}{1-\frac{(1-\varpi)}{1+\rho \hat{c}^{\prime}(1)}-\frac{\varpi}{1+(1-1 / \varpi) \rho \hat{c}^{\prime}(1)}} \\
& =\frac{1+\rho \hat{c}^{\prime}(1)}{\rho \hat{c}^{\prime}(1)}=\frac{-1+\frac{b}{1+\rho(1-b) \hat{c}^{\prime}(1)}}{-1+\frac{1}{1+\rho(1-b) \hat{c}^{\prime}(1)}} \\
& =\frac{-1+b \hat{u}^{L}}{-1+\hat{u}^{L}}=\frac{r H-r b u^{L}}{(r+\pi) L-\pi H-r u^{L}} .
\end{aligned}
$$

Hence when $L=H, p$ is $\left((r+\pi) L-\pi H-r u^{L}\right) /\left((1-\varpi) L_{\mathcal{L}}+\varpi L_{\mathcal{H}}-L\right)=$ $\left(r H-r b u^{L}\right) /\left(H_{\mathcal{H}}-H\right)>0$, which implies $d L / d t=d H / d t=0$. The state is stationary after $N$ and before auditing arrives.

Proof of Lemma 8. Given the initial promised utilities $(L, H)$, we need to verify that

(i) the cost of the contract implied by $\hat{c}^{x^{* *}}(\cdot)$ is $C(L, H)$

(ii) the costs of other I.C. contracts are weakly higher than $C(L, H)$.

We verify only (ii) here, since the proof for (i) can be obtained simply by replacing the following inequalities with equalities.

Suppose that $\left\{\left(\tilde{c}^{H}(t), \tilde{c}^{L}(t), \tilde{p}(t)\right) ; t \geq 0\right\}$ is an I.C. contract. The proof that shows (26) in Section 2.2.2 can be used to prove that

$$
\begin{aligned}
C(L, H) \leq \int_{0}^{\infty} e^{-(r+\pi) t-\int_{0}^{t} p(\xi) d \xi}\left(\pi c(\tilde{H}(t))+r \tilde{c}^{L}(t)\right. \\
\left.\quad+p(t)\left((1-\varpi) C\left(\tilde{L}_{\mathcal{L}}(t), \tilde{H}_{\mathcal{L}}(t)\right)+\varpi C\left(\tilde{L}_{\mathcal{H}}(t), \tilde{H}_{\mathcal{H}}(t)\right)+\gamma\right)\right) d t,
\end{aligned}
$$

which can be rewritten equivalently as

$$
\begin{aligned}
C(L, H) \leq E\left[\int_{0}^{\tau_{1}} e^{-(r+\pi) t}\left(\pi c(\tilde{H}(t))+r \tilde{c}^{L}(t)\right) d t\right] & \\
& +E\left[e^{-r \tau_{1}} \gamma\right]+E\left[e^{-r \tau_{1}} C\left(\tilde{L}\left(\tau_{1}\right), \tilde{H}\left(\tau_{1}\right)\right)\right],
\end{aligned}
$$

where $\tau_{1}$ is the first auditing time and $\left(\tilde{L}\left(\tau_{1}\right), \tilde{H}\left(\tau_{1}\right)\right)$ is the after-auditing state vector contingent on the signal at $\tau_{1}$ (i.e., it is either $\left(\tilde{L}_{\mathcal{L}}, \tilde{H}_{\mathcal{L}}\right)$ or $\left.\left(\tilde{L}_{\mathcal{H}}, \tilde{H}_{\mathcal{H}}\right)\right)$. Inductively, we obtain

$$
\begin{aligned}
C(L, H) \leq E\left[\int_{0}^{\tau_{n}} e^{-(r+\pi) t}(\pi c(\tilde{H}(t))+\right. & \left.\left.r \tilde{c}^{L}(t)\right) d t\right] \\
& +E\left[\sum_{i=1}^{n} e^{-r \tau_{i}} \gamma\right]+E\left[e^{-r \tau_{n}} C\left(\tilde{L}\left(\tau_{n}\right), \tilde{H}\left(\tau_{n}\right)\right)\right],
\end{aligned}
$$


where $\tau_{n}$ is the $n$th auditing time. Without loss of generality, we may assume that $\lim _{n \rightarrow \infty} \tau_{n}=\infty$ almost surely (otherwise, the principal audits infinitely many times in finite time and the auditing cost is infinity). Hence taking limit $n \rightarrow \infty$ in the above inequality and applying Lemma 11 yield

$$
C(L, H) \leq E\left[\int_{0}^{\infty} e^{-(r+\pi) t}\left(\pi c(\tilde{H}(t))+r \tilde{c}^{L}(t)\right) d t\right]+E\left[\sum_{i=1}^{\infty} e^{-r \tau_{i}} \gamma\right] .
$$

Proof of Lemma 9. If $H / L \leq x^{* *}$, it is easy to show that $M(P)>C(L, H)$ because

$$
\begin{aligned}
P((1-\varpi) C & \left.\left(L_{\mathcal{L}}, H_{\mathcal{L}}\right)+\varpi C\left(L_{\mathcal{H}}, H_{\mathcal{H}}\right)+\gamma\right)+(1-P) C(\bar{L}, \bar{H}) \\
& >P\left((1-\varpi) C\left(L_{\mathcal{L}}, x^{* *} L_{\mathcal{L}}\right)+\varpi C\left(L_{\mathcal{H}}, x^{* *} L_{\mathcal{H}}\right)+\gamma\right)+(1-P) C\left(\bar{L}, x^{* *} \bar{L}\right) \\
& \geq C(L, H)+P \gamma .
\end{aligned}
$$

In the rest of the proof, we assume that $H / L>x^{* *}$. Because $C(L, H)$ is strictly convex in $\left\{(L, H): L<0, x^{* *} \leq H / L \leq 1\right\}$, the optimal solution to (21) is unique and is denoted as $L_{\mathcal{L}}(P), H_{\mathcal{L}}(P), L_{\mathcal{H}}(P), H_{\mathcal{H}}(P), \bar{L}(P), \bar{H}(P)$.

First, we show that the objective $M(P)$ is a strictly convex function of $P$. Suppose $\theta \in(0,1)$ and $P=\theta P_{1}+(1-\theta) P_{2}, P_{1} \neq P_{2}$. Then construct a solution for the problem $M(P)$ as

$$
\begin{aligned}
\left(\tilde{L}_{\mathcal{L}}, \tilde{H}_{\mathcal{L}}\right) & =\frac{\theta P_{1}}{\theta P_{1}+(1-\theta) P_{2}}\left(L_{\mathcal{L}}\left(P_{1}\right), H_{\mathcal{L}}\left(P_{1}\right)\right)+\frac{(1-\theta) P_{2}}{\theta P_{1}+(1-\theta) P_{2}}\left(L_{\mathcal{L}}\left(P_{2}\right), H_{\mathcal{L}}\left(P_{2}\right)\right) \\
\left(\tilde{L}_{\mathcal{H}}, \tilde{H}_{\mathcal{H}}\right) & =\frac{\theta P_{1}}{\theta P_{1}+(1-\theta) P_{2}}\left(L_{\mathcal{H}}\left(P_{1}\right), H_{\mathcal{H}}\left(P_{1}\right)\right)+\frac{(1-\theta) P_{2}}{\theta P_{1}+(1-\theta) P_{2}}\left(L_{\mathcal{H}}\left(P_{2}\right), H_{\mathcal{H}}\left(P_{2}\right)\right) \\
(\bar{L}, \bar{H}) & =\frac{\theta\left(1-P_{1}\right)}{\theta\left(1-P_{1}\right)+(1-\theta)\left(1-P_{2}\right)}\left(\bar{L}\left(P_{1}\right), \bar{H}\left(P_{1}\right)\right) \\
& +\frac{(1-\theta)\left(1-P_{2}\right)}{\theta\left(1-P_{1}\right)+(1-\theta)\left(1-P_{2}\right)}\left(\bar{L}\left(P_{2}\right), \bar{H}\left(P_{2}\right)\right) .
\end{aligned}
$$

The strict convexity of $C(L, H)$ implies that

$$
\begin{aligned}
M(P) \leq P\left((1-\varpi) C\left(\tilde{L}_{\mathcal{L}}, \tilde{H}_{\mathcal{L}}\right)+\varpi C\left(\tilde{L}_{\mathcal{H}}, \tilde{H}_{\mathcal{H}}\right)+\gamma\right)+(1-P) C(\bar{L}, \bar{H}) \\
<P(1-\varpi)\left(\frac{\theta P_{1}}{\theta P_{1}+(1-\theta) P_{2}} C\left(L_{\mathcal{L}}\left(P_{1}\right), H_{\mathcal{L}}\left(P_{1}\right)\right)\right. \\
\left.\quad+\frac{(1-\theta) P_{2}}{\theta P_{1}+(1-\theta) P_{2}} C\left(L_{\mathcal{L}}\left(P_{2}\right), H_{\mathcal{L}}\left(P_{2}\right)\right)\right) \\
\quad+P \varpi\left(\frac{\theta P_{1}}{\theta P_{1}+(1-\theta) P_{2}} C\left(L_{\mathcal{H}}\left(P_{1}\right), H_{\mathcal{H}}\left(P_{1}\right)\right)\right. \\
\left.+\frac{(1-\theta) P_{2}}{\theta P_{1}+(1-\theta) P_{2}} C\left(L_{\mathcal{H}}\left(P_{2}\right), H_{\mathcal{H}}\left(P_{2}\right)\right)\right) \\
+(1-P)\left(\frac{\theta\left(1-P_{1}\right)}{\theta\left(1-P_{1}\right)+(1-\theta)\left(1-P_{2}\right)} C\left(\bar{L}\left(P_{1}\right), \bar{H}\left(P_{1}\right)\right)\right.
\end{aligned}
$$




$$
\begin{aligned}
& \left.\quad+\frac{(1-\theta)\left(1-P_{2}\right)}{\theta\left(1-P_{1}\right)+(1-\theta)\left(1-P_{2}\right)} C\left(\bar{L}\left(P_{2}\right), \bar{H}\left(P_{2}\right)\right)\right)+P \gamma \\
& =\theta\left(P_{1}\left((1-\varpi) C\left(L_{\mathcal{L}}\left(P_{1}\right), H_{\mathcal{L}}\left(P_{1}\right)\right)+\varpi C\left(L_{\mathcal{H}}\left(P_{1}\right), H_{\mathcal{H}}\left(P_{1}\right)\right)+\gamma\right)\right. \\
& \left.\quad+\left(1-P_{1}\right) C\left(\bar{L}\left(P_{1}\right), \bar{H}\left(P_{1}\right)\right)\right) \\
& \quad+(1-\theta)\left(P_{2}\left((1-\varpi) C\left(L_{\mathcal{L}}\left(P_{2}\right), H_{\mathcal{L}}\left(P_{2}\right)\right)+\varpi C\left(L_{\mathcal{H}}\left(P_{2}\right), H_{\mathcal{H}}\left(P_{2}\right)\right)+\gamma\right)\right. \\
& \left.\quad+\left(1-P_{2}\right) C\left(\bar{L}\left(P_{2}\right), \bar{H}\left(P_{2}\right)\right)\right) \\
& =\theta M\left(P_{1}\right)+(1-\theta) M\left(P_{2}\right) .
\end{aligned}
$$

Second, we show that $M(P)>C(L, H)=M(0)$ for all $P>0$. Because $M(P)$ is strictly convex, it is sufficient to prove that $M^{\prime}(0) \geq 0$. To finish the proof, we show that

$$
M^{\prime}(0)=\gamma-\mathcal{M}\left(\hat{c}^{\prime}(x), x\right) \geq 0, \quad \text { where } x=\frac{H}{L} .
$$

Denote the Lagrangian multipliers on constraints (22) and (23) as $\xi_{L}(P)$ and $\xi_{H}(P)$, respectively. Then the first-order conditions are

$$
\begin{aligned}
M^{\prime}(P)= & \left((1-\varpi) C\left(L_{\mathcal{L}}(P), H_{\mathcal{L}}(P)\right)+\varpi C\left(L_{\mathcal{H}}(P), H_{\mathcal{H}}(P)\right)+\gamma-C(\bar{L}(P), \bar{H}(P))\right) \\
& \quad-\left(\xi_{L}(P)\left((1-\varpi) L_{\mathcal{L}}(P)+\varpi L_{\mathcal{H}}(P)-\bar{L}(P)\right)+\xi_{H}(P)\left(H_{\mathcal{H}}(P)-\bar{H}(P)\right)\right) \\
\xi_{L}(P)= & C_{L}\left(L_{\mathcal{L}}(P), H_{\mathcal{L}}(P)\right)=C_{L}\left(L_{\mathcal{H}}(P), H_{\mathcal{H}}(P)\right)=C_{L}(\bar{L}(P), \bar{H}(P)) \\
0= & C_{H}\left(L_{\mathcal{L}}(P), H_{\mathcal{L}}(P)\right) \\
\frac{\xi_{H}(P)}{\varpi}= & C_{H}\left(L_{\mathcal{H}}(P), H_{\mathcal{H}}(P)\right) \\
\xi_{H}(P)= & C_{H}(\bar{L}(P), \bar{H}(P)) .
\end{aligned}
$$

Since $\lim _{P \rightarrow 0} \bar{L}(P)=L, \lim _{P \rightarrow 0} \bar{H}(P)=H$, these first-order conditions imply

$$
\begin{aligned}
\lim _{P \downarrow 0} M^{\prime}(P)= & \left((1-\varpi) C\left(L_{\mathcal{L}}, H_{\mathcal{L}}\right)+\varpi C\left(L_{\mathcal{H}}, H_{\mathcal{H}}\right)+\gamma-C(L, H)\right) \\
& -\left(C_{L}(L, H)\left((1-\varpi) L_{\mathcal{L}}+\varpi L_{\mathcal{H}}-L\right)+C_{H}(L, H)\left(H_{\mathcal{H}}-H\right)\right) \\
= & \gamma-\mathcal{M}\left(\hat{c}^{\prime}(x), x\right),
\end{aligned}
$$

where the second equality follows from (32).

Lemma 10. When $H>L$, the trajectories implied by the candidate solution satisfy $d L / d t<0, d H / d t<0, d C_{L} / d t=0$, and $d C_{H} / d t<0$.

Proof. (i) Because $x<1, \hat{u}^{L}=1 /\left(1+\hat{c}^{\prime}(x) \rho(x-b)\right)<1$, and $L<0$, then $d L / d t=$ $\left(r+\pi-\pi x-r \hat{u}^{L}\right) L<0$.

(ii) Because $L<0$ decreases and $x>0$ increases with $t$, then $H=L x$ decreases with $t$. 
(iii) It follows from $C(L, H)=c(L)+\hat{c}(x)$ that $C_{H}=\hat{c}^{\prime}(x) / L, C_{L}=-1 /(\rho L)-$ $\hat{c}^{\prime}(x) x / L$. Hence

$$
\begin{aligned}
\frac{d C_{H}}{d t}= & \frac{\frac{d \hat{c}^{\prime}(x)}{d t} L-\hat{c}^{\prime}(x)\left(r+\pi-\pi x-r \hat{u}^{L}\right) L}{L^{2}} \\
= & \left(\left(\frac{\pi}{\rho}\left(\frac{1}{x}-1\right)+\hat{c}^{\prime}(x)\left(r+2 \pi-r \hat{u}^{L}-2 \pi x\right)\right) L\right. \\
& \left.\quad-\hat{c}^{\prime}(x)\left(r+\pi-\pi x-r \hat{u}^{L}\right) L\right) / L^{2} \\
= & \frac{\pi(1-x)}{L}\left(\frac{1}{\rho x}+\hat{c}^{\prime}(x)\right) \\
< & 0 .
\end{aligned}
$$

(iv) Because $C_{L}=-1 /(\rho L)-C_{H} x$, we have

$$
\begin{aligned}
\frac{d C_{L}}{d t} & =\frac{\left(r+\pi-\pi x-r \hat{u}^{L}\right) L}{\rho L^{2}}-C_{H}\left(r(x-b) \hat{u}^{L}+\pi(x-1) x\right)-\frac{d C_{H}}{d t} x \\
& =\frac{r+\pi-\pi x-r \hat{u}^{L}-\rho \hat{c}^{\prime}(x)\left(r(x-b) \hat{u}^{L}+\pi(x-1) x\right)}{\rho L} \\
& \quad-\frac{\pi(1-x)}{L}\left(\frac{1}{\rho x}+\hat{c}^{\prime}(x)\right) x \\
& =0 .
\end{aligned}
$$

Lemma 11. (i) In the model with no auditing, if the cost of an I.C. contract $\left\{\left(c^{H}(t)\right.\right.$, $\left.\left.c^{L}(t)\right) ; t \geq 0\right\}$ is finite, then

$$
\lim _{T \rightarrow \infty} e^{-(r+\pi) T} C(L(T), H(T))=0 .
$$

(ii) In the model with auditing, if the cost of an I.C. contract $\left\{\left(c^{H}(t), c^{L}(t), p(t)\right) ; t \geq 0\right\}$ is finite, then

$$
\lim _{T \rightarrow \infty} E\left[e^{-r T} C(L(T), H(T))\right]=0 .
$$

Proof. The proof for part (ii) is similar to part (i), but much simpler because the distortion function $\hat{c}^{x^{* *}}(\cdot)$ is bounded with auditing. We only prove part (i).

Recall that the upper bound for $\hat{c}^{x^{*}}(\cdot)$ is

$$
\begin{aligned}
& \mathscr{H}(\bar{d}(x), x) \\
& =\frac{1}{r+\pi}\left(-\frac{1}{\rho}\left(r+\pi-\pi x-r \hat{u}^{L}\right)+r c\left(-\hat{u}^{L}\right)+\pi c(-x)\right) \\
& =\frac{1}{r+\pi}\left(-\frac{1}{\rho}\left(r+\pi-\pi x-r \hat{u}^{L}\right)+r c\left(-\frac{\pi(1-x) b}{r(x-b)}\right)+\pi c(-x)\right)
\end{aligned}
$$




$$
\begin{aligned}
=\frac{1}{r+\pi} & \left(-\frac{1}{\rho}\left(r+\pi-\pi x-r \hat{u}^{L}\right)+r c\left(-\frac{\pi b}{r(x-b)}\right)+\pi c(-x)\right) \\
& -\frac{r}{\rho(r+\pi)} \log (1-x) .
\end{aligned}
$$

There is a lower bound for the costs of all I.C. contracts delivering $(-1,-x)$. Since all such contracts satisfy

$$
\begin{aligned}
& -1=\int_{0}^{\infty} e^{-(r+\pi) t}\left(\pi H(t)+r u^{L}(t)\right) d t \\
& -x \geq \int_{0}^{\infty} e^{-(r+\pi) t}\left(\pi H(t)+r b u^{L}(t)\right) d t,
\end{aligned}
$$

we may solve a relaxed problem in which only (33) and (34) are imposed. The cost $\underline{c}(x)$ from this problem serves as a lower bound. If $x<(\pi+r b) /(r+\pi)$, the constraint (34) is slack and

$$
H(t)=u^{L}(t)=-1 \quad \forall t \geq 0 .
$$

Otherwise, (34) binds and

$$
H(t)=\frac{r+\pi}{\pi} \frac{b-x}{1-b}, \quad u^{L}(t)=\frac{r+\pi}{r} \frac{x-1}{1-b} \quad \forall t \geq 0 .
$$

Hence when $x \in[(\pi+r b) /(r+\pi), 1)$,

$$
\begin{aligned}
\underline{c}(x) & =\frac{\pi}{r+\pi} c\left(\frac{r+\pi}{\pi} \frac{b-x}{1-b}\right)+\frac{r}{r+\pi} c\left(\frac{r+\pi}{r} \frac{x-1}{1-b}\right) \\
& =\frac{\pi}{r+\pi} c\left(\frac{r+\pi}{\pi} \frac{b-x}{1-b}\right)+\frac{r}{r+\pi} c\left(\frac{r+\pi}{r} \frac{-1}{1-b}\right)-\frac{r}{\rho(r+\pi)} \log (1-x) .
\end{aligned}
$$

It follows from the two bounds that $\mathscr{H}(\bar{d}(x), x)-\underline{c}(x)$ is bounded by some $B>0$.

For any I.C. contract with finite cost, the promise-keeping constraint and finite cost imply that

$$
\begin{aligned}
\lim _{T \rightarrow \infty} e^{-(r+\pi) T} L(T) & =0 \\
\lim _{T \rightarrow \infty} \int_{T}^{\infty} e^{-(r+\pi) t}\left(\pi c(H(t))+r c^{L}(t)\right) d t & =0 .
\end{aligned}
$$

Then,

$$
\begin{aligned}
\lim _{T \rightarrow \infty} e^{-(r+\pi) T}\left(c(L(T))+\hat{c}^{x^{*}}(x(T))\right) \\
\quad \leq \lim _{T \rightarrow \infty} e^{-(r+\pi) T}(c(L(T))+\mathcal{H}(\bar{d}(x), x)) \\
\leq \lim _{T \rightarrow \infty} e^{-(r+\pi) T}(c(L(T))+\underline{c}(x(T))+B) \\
\quad \leq \lim _{T \rightarrow \infty} \int_{T}^{\infty} e^{-(r+\pi) t}\left(\pi c(H(t))+r c^{L}(t)\right) d t+\lim _{T \rightarrow \infty} e^{-(r+\pi) T} B=0,
\end{aligned}
$$


where the second inequality follows from the choice of $B$ and the third inequality follows from the fact that $c(L(T))+\underline{c}(x(T))$ is a lower bound for the cost of the continuation contract that delivers $(L(T), L(T) x(T))$. On the other hand,

$$
\begin{aligned}
\lim _{T \rightarrow \infty} e^{-(r+\pi) T}\left(c(L(T))+\hat{c}^{x^{*}}(x(T))\right) & \geq \lim _{T \rightarrow \infty} e^{-(r+\pi) T} c(L(T)) \\
& \geq \lim _{T \rightarrow \infty} e^{-(r+\pi) T} \frac{L(T)}{\rho}=0 .
\end{aligned}
$$

We conclude that $\lim _{T \rightarrow \infty} e^{-(r+\pi) T} C(L(T), H(T))=0$.

Lemma 12. In the model with no auditing, if the cost of an I.C. contract $\left\{\left(c^{H}(t), c^{L}(t)\right)\right.$; $t \geq 0\}$ is finite, then

$$
X(T) \leq C(L(T), H(T)) \quad \text { for all } T \geq 0,
$$

where

$$
\begin{aligned}
X(T) \equiv C(L(0), H(0))+\int_{0}^{T} C_{L}(L(t), H(t)) & \left((r+\pi) L(t)-\pi H(t)-r u^{L}(t)\right) d t \\
& +\int_{0}^{T} C_{H}(L(t), H(t))\left(r H(t)-r b u^{L}(t)\right) d t .
\end{aligned}
$$

Proof. Fix a $T>0$. First we find a compact subset $G$ of the domain of the cost function that contains the path $\{(L(t), H(t)): 0 \leq t \leq T\}$. The promise-keeping constraint implies that $L(\cdot)$ is continuous in $t$. The finite cost of the contract implies that $\max _{0 \leq t \leq T} x(t)<1$, because otherwise the lower bound in Lemma 11 implies that the cost is infinity. Define

$$
G \equiv\left\{(l, h) \in \mathbb{R}^{2}: \min _{0 \leq t \leq T} L(t) \leq l \leq \max _{0 \leq t \leq T} L(t), 0 \leq \frac{h}{l} \leq \max _{0 \leq t \leq T} x(t)\right\} .
$$

Because $C_{L}(\cdot, \cdot)$ and $C_{H}(\cdot, \cdot)$ are continuous functions, let $M>0$ be an upper bound for them on the compact set $G$. Uniform continuity on $G$ states that for any $\epsilon>0$, there exists a $\delta>0$, such that if $\left|\left(l_{1}, h_{1}\right)-\left(l_{2}, h_{2}\right)\right| \leq \delta$, then

$$
\left|C_{L}\left(l_{1}, h_{1}\right)-C_{L}\left(l_{2}, h_{2}\right)\right| \leq \epsilon, \quad\left|C_{H}\left(l_{1}, h_{1}\right)-C_{H}\left(l_{2}, h_{2}\right)\right| \leq \epsilon .
$$

Second, we show that both $L(\cdot)$ and $H(\cdot)$ are of bounded variation on $[0, T]$. The function $L(\cdot)$ is absolutely continuous, hence it has bounded variation. Incentive constraints imply that

$$
V^{+}(T)=\sup \left\{\sum_{i=1}^{n-1}\left(H\left(t_{i+1}\right)-H\left(t_{i}\right)\right)^{+}:\left\{t_{1}, \ldots, t_{n}\right\} \text { is a partition of }[0, T]\right\}
$$

is finite. Because $H(\cdot)$ is a bounded function, it follows from $H(T)-H(0)=V^{+}(T)-$ $V^{-}(T)$ that $V^{-}(T)$ is also finite. Hence $H(\cdot)$ has bounded variation.

Third, we choose a partition $\left\{t_{1}, \ldots, t_{n}\right\}, 0=t_{1}<t_{2}<\cdots<t_{n}=T$ of $[0, T]$ that is fine enough such that in any interval $\left[t_{i}, t_{i+1}\right]$, either the total variations of $L(\cdot)$ and $H(\cdot)$ are 
less than $\delta$ (then $\left[t_{i}, t_{i+1}\right]$ is called a type 1 interval) or $H(\cdot)$ has a discrete jump larger than $\delta$ (called a type 2 interval). Furthermore, the total variation excluding the discrete jumps in all the type 2 intervals can be made less than $\epsilon$. Then

$$
\begin{aligned}
C(L(T), H(T))- & C(L(0), H(0)) \\
& =\sum_{i=1}^{n-1} C\left(L\left(t_{i+1}\right), H\left(t_{i+1}\right)\right)-C\left(L\left(t_{i}\right), H\left(t_{i}\right)\right) \\
& =\sum_{i=1}^{n-1} C_{L}\left(L_{i}, H_{i}\right)\left(L\left(t_{i+1}\right)-L\left(t_{i}\right)\right)+C_{H}\left(L_{i}, H_{i}\right)\left(H\left(t_{i+1}\right)-H\left(t_{i}\right)\right),
\end{aligned}
$$

where $L_{i} \in\left[L\left(t_{i}\right), L\left(t_{i+1}\right)\right]$ and $H_{i} \in\left[H\left(t_{i}\right), H\left(t_{i+1}\right)\right]$ exist because of the mean value theorem. Hence

$$
\begin{aligned}
& \sum_{i=1}^{n-1} C_{L}\left(L_{i}, H_{i}\right)\left(L\left(t_{i+1}\right)-L\left(t_{i}\right)\right)+C_{H}\left(L_{i}, H_{i}\right)\left(H\left(t_{i+1}\right)-H\left(t_{i}\right)\right) \\
& \geq \sum_{i \in \text { type } 1} \int_{t_{i}}^{t_{i+1}}\left(C_{L}\left(L_{i}, H_{i}\right) d L(t)+C_{H}\left(L_{i}, H_{i}\right)\left(r H(t)-r b u^{L}(t)\right) d t\right)-2 M \epsilon \\
& \geq \sum_{i \in \operatorname{type} 1} \int_{t_{i}}^{t_{i+1}}\left(\sup _{t \in\left[t_{i}, t_{i+1}\right]} C_{L}(L(t), H(t))-\epsilon\right) d L(t) \\
& \left.\quad+\left(\sup _{t \in\left[t_{i}, t_{i+1}\right]} C_{H}(L(t), H(t))-\epsilon\right)\left(r H(t)-r b u^{L}(t)\right) d t\right)-2 M \epsilon \\
& \geq \sum_{i=1}^{n-1} \int_{t_{i}}^{t_{i+1}}\left(\left(\sup _{t \in\left[t_{i}, t_{i+1}\right]} C_{L}(L(t), H(t))-\epsilon\right) d L(t)\right. \\
& \left.\quad+\left(\sup _{t \in\left[t_{i}, t_{i+1}\right]} C_{H}(L(t), H(t))-\epsilon\right)\left(r H(t)-r b u^{L}(t)\right) d t\right)-2 M \epsilon-2(M+\epsilon) \epsilon \\
& \geq \int_{0}^{T}\left(C_{L}(L(t), H(t)) d L(t)+C_{H}(L(t), H(t))\left(r H(t)-r b u^{L}(t)\right) d t\right) \\
& \quad-\epsilon(L(T)-L(0))-\epsilon \int_{0}^{T}\left(r H(t)-r b u^{L}(t)\right) d t-2 M \epsilon-2(M+\epsilon) \epsilon .
\end{aligned}
$$

Since the inequality holds for all $\epsilon>0$, (35) is true.

Lemma 13. Assume CRRA preferences and the same auditing technology as in Section 3. The optimal auditing probability is close to zero when $L_{0}$ is either extremely high or extremely low.

Proof. When $L_{0}$ is high, consider a no-auditing I.C. contract in which $c^{H}(t)$ and $c^{L}(t)$ are independent of $t, c^{H}-c^{L}=w_{H}-w_{L}$, and

$$
\frac{r u\left(c^{L}\right)+\pi u\left(c^{H}\right)}{r+\pi}=L_{0} .
$$


The difference between the cost of this contract, $\left(r c^{L}+\pi c^{H}\right) /(r+\pi)$, and that of the full-information constant consumption, $c\left(L_{0}\right)$, is the risk premium, which can be easily verified to approach zero when $L_{0} \rightarrow \infty$ because the absolute risk aversion approaches zero. Hence the principal does not need to audit with large probability when $L_{0}$ is large.

The lowest utility that the principal can deliver to the agent is $\underline{L} \equiv(r u(0)+$ $\left.\pi u\left(w_{H}-w_{L}\right)\right) /(r+\pi)$. This is because the agent's consumption must be nonnegative and, hence, the high-income agent can consume at least $w_{H}-w_{L}$ by underreporting.

When $L_{0}$ is close to $\underline{L}$, consider again the above no-auditing I.C. contract in which $c^{H}-c^{L}=w_{H}-w_{L}$ and

$$
\frac{r u\left(c^{L}\right)+\pi u\left(c^{H}\right)}{r+\pi}=L_{0} .
$$

Because $c^{L} \approx 0$, the cost of this no-auditing contract, $\left(r c^{L}+\pi c^{H}\right) /(r+\pi)$, is close to $\pi\left(w_{H}-w_{L}\right) /(r+\pi)$, which is a lower bound for the costs of all I.C. contracts. To see that $\pi\left(w_{H}-w_{L}\right) /(r+\pi)$ is a lower bound, note that the high-income agent's continuation utility must be greater than or equal to $u\left(w_{H}-w_{L}\right)$, as the low-income agent's consumption must be nonnegative. Hence the principal does not need to audit with large probability when $L_{0}$ is close to $\underline{L}$.

\section{REFERENCES}

Aliprantis, Charalambos D. and Owen Burkinshaw (1990), Principles of Real Analysis, second edition. Academic Press, San Diego. [267]

Armenter, Roc and Thomas M. Mertens (2010), "Fraud deterrence in dynamic Mirrleesian economies.” Working Paper 10-7, Federal Reserve Board of Philadelphia. [266]

Becker, Gary S. (1968), "Crime and punishment: An economic approach.” Journal of Political Economy, 76, 169-217. [241]

Fernandes, Ana and Christopher Phelan (2000), "A recursive formulation for repeated agency with history dependence." Journal of Economic Theory, 91, 223-247. [242, 247]

Golosov, Mikhail and Aleh Tsyvinski (2006), "Designing optimal disability insurance: A case for asset testing." Journal of Political Economy, 114, 257-279. [245]

Green, Edward J. (1987), “Lending and the smoothing of uninsurable income." In Contractual Arrangements for Intertemporal Trade (Edward C. Prescott and Neil Wallace, eds.), 3-25, University of Minnesota Press, Minneapolis. [243, 248, 253, 254, 261]

Hopenhayn, Hugo and Juan P. Nicolini (1997), “Optimal unemployment insurance." Journal of Political Economy, 105, 412-438. [245]

Kapicka, Marek (2010), "Efficient allocations in dynamic private information economies with persistent shocks: A first order approach.” Unpublished paper, University of California, Santa Barbara. [265]

Monnet, Cyril and Erwan Quintin (2005), "Optimal contracts in a dynamic costly state verification model.” Economic Theory, 26, 867-885. [244, 262] 
Nakamura, Hisashi (2009), "A dynamic theory of debt restructuring." Unpublished paper, University of Tokyo. [261]

Popov, Latchezar (2007), "Stochastic costly state verification and dynamic contracts." Unpublished paper, University of Iowa. [244, 264]

Ravikumar, B. and Yuzhe Zhang (2010), "Optimal auditing in a dynamic model of tax compliance." Unpublished paper, University of Iowa. [264]

Reinganum, Jennifer F. and Louis L. Wilde (1985), "Income tax compliance in a principal-agent framework.” Journal of Public Economics, 26, 1-18. [241]

Reinganum, Jennifer F. and Louis L. Wilde (1986), "Equilibrium verification and reporting policies in a model of tax compliance." International Economic Review, 27, 739-760. [241]

Rogerson, William P. (1985), "Repeated moral hazard." Econometrica, 53, 69-76. [253]

Stigler, George J. (1970), “The optimum enforcement of laws.” Journal of Political Economy, 78, 526-536. [241]

Townsend, Robert M. (1979), "Optimal contracts and competitive markets with costly state verification.” Journal of Economic Theory, 21, 265-293. [241]

Wang, Cheng (2005), “Dynamic costly state verification.” Economic Theory, 25, 887-916. $[243,244,261,262]$

Williams, Noah (2011), “Persistent private information.” Econometrica, 79, 1233-1275. [265]

Zhang, Yuzhe (2009), "Dynamic contracting with persistent shocks." Journal of Economic Theory, 144, 635-675. [242, 247]

Submitted 2010-2-24. Final version accepted 2011-5-2. Available online 2011-5-8. 